\title{
Temperature quenching in LAB based liquid scintillator
}

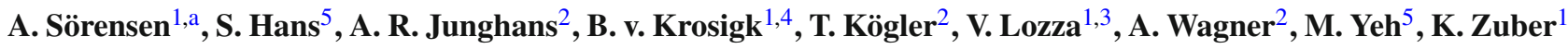 \\ ${ }^{1}$ Institute for Nuclear- and Particle Physics, Technische Universität Dresden, 01069 Dresden, Germany \\ ${ }^{2}$ Helmholtz-Zentrum Dresden-Rossendorf, 01314 Dresden, Germany \\ ${ }^{3}$ Present address: Laboratório de Instrumentação e Física Experimental de Partículas, 1649-003 Lisboa, Portugal \\ ${ }^{4}$ Department of Physics and Astronomy, University of British Columbia, Vancouver, BC V6T 1z1, Canada \\ ${ }^{5}$ Chemistry Devision, Brookhaven National Laboratory, Upton, NY 11973-500, USA
}

Received: 18 October 2017 / Accepted: 18 December 2017 / Published online: 4 January 2018

(C) The Author(s) 2018. This article is an open access publication

\begin{abstract}
The effect of temperature changes on the light output of LAB based liquid scintillator is investigated in a range from -5 to $30^{\circ} \mathrm{C}$ with $\alpha$-particles and electrons in a small scale setup. Two PMTs observe the scintillator liquid inside a cylindrically shaped aluminum cuvette that is heated or cooled and the temperature dependent PMT sensitivity is monitored and corrected. The $\alpha$-emitting isotopes in dissolved radon gas and in natural Samarium (bound to a LAB solution) excite the liquid scintillator mixtures and changes in light output with temperature variation are observed by fitting light output spectra. Furthermore, also changes in light output by compton electrons, which are generated from external calibration $\gamma$-ray sources, is analysed with varying temperature. Assuming a linear behaviour, a combined negative temperature coefficient of $(-0.29 \pm 0.01) \% /{ }^{\circ} \mathrm{C}$ is found. Considering hints for a particle type dependency, electrons show $(-0.17 \pm 0.02) \% /{ }^{\circ} \mathrm{C}$, whereas the temperature dependency seems stronger for $\alpha$-particles, with $(-0.35 \pm 0.03) \% /{ }^{\circ} \mathrm{C}$. Due to a high sampling rate, a pulse shape analysis can be performed and shows an enhanced slow decay component at lower temperatures, pointing to reduced non-radiative triplet state de-excitations.
\end{abstract}

\section{Introduction}

In neutrino- and astroparticle physics, liquid scintillator detectors have been used in many milestone experiments, and upcoming large-scale liquid scintillator experiments also indicate the importance of this detector material. Linear alkyl benzene $\left(\mathrm{LAB}^{1}\right)$ as solvent together with $\mathrm{PPO}^{2}$ as primary solute are the basis for a modern organic liquid scintillator,

\footnotetext{
a e-mail: arnd.soerensen@gmail.com

${ }^{1} \mathrm{C}_{6} \mathrm{H}_{5} \mathrm{C}_{n} \mathrm{H}_{2 n+1}, n=10-13$.

2 2,5-diphenyloxazole.
}

which is already in use by the Daya-Bay [1] and RENO [2] experiments, will soon be used by $\mathrm{SNO}+[3]$ and is also foreseen for future neutrino detectors like JUNO [4].

While most properties of LAB based liquid scintillators are well studied, the influence of temperature on the light generation is poorly known and only recently interest in this matter emerged again. The SNO+ experiment will be operated at $\approx 12^{\circ} \mathrm{C}$ due to the cooled water shielding surrounding the liquid scintillator vessel [3], and JUNO investigated the eventually positive aspects of a significant cooling of the active detector medium on the energy resolution [5].

Previous works On the subject of temperature effects in liquid scintillators, real data and good studies are scarce, especially when focusing on changes in light yield. The very first investigations were carried out by Seliger and Ziegler [6] in the mid 1950s. Unfortunately the temperature of the whole measurement setup was changed, including the PMTs. Therefore that work was critically discussed and commonly disqualified by Laustriat, Coche $[7,8]$ and Birks [9].

The former two did investigate temperature effects themselves with three different toluene solutions. While maintaining the PMTs at constant temperature and exciting the mixture via UV light, negative temperature coefficients in light output for PPO at about $20 \%$ and about $10 \%$ for PBD are reported, both at $-10{ }^{\circ} \mathrm{C}$. When ${ }^{137} \mathrm{Cs}$ was used as a radiation source, the effect is much less expressed with less than $5 \%$ for both solvents, again at $-10^{\circ} \mathrm{C}$. This study is difficult to interpret quantitatively since the setup is not described, it is not explained how temperature stability on the PMTs is achieved or how this was controlled, and no uncertainties are reported. Therefore, unfortunately, this can only be seen as an indication that scintillation light output might be quenched at higher temperatures and is enhanced when the liquid is beeing cooled down. 
More than two decades later Homma, Murase and Sonehara [10-12] revisited this topic and carried out some measurements with $\alpha$-and $\beta$-radiation sources dissolved in five different liquid scintillator solvents: o-xylene, m-xylene, ethylbenzene, benzene and cumene. The single fluor solute in all of them was $4 \mathrm{~g} / 1 \mathrm{PPO}$. The radiation sources were ${ }^{241} \mathrm{Am}$ and ${ }^{131 \mathrm{~m}} \mathrm{Xe}$, which is, besides $\gamma$-rays and $\mathrm{X}$-rays, emitting mono-energetic conversion electrons of $E_{\beta}=0.164 \mathrm{MeV}$. Even though pulse-height spectra are shown, again the data is not analysed quantitatively, no uncertainties are given and only a very rough description of the setup is included. It is claimed that the PMT temperature is somehow stabilised for all measurements. In a very coarse 'by hand' approximation of presented energy spectra, a temperature coefficient of about $-0.5 \% /{ }^{\circ} \mathrm{C}$ might be extracted. Nevertheless for all scintillator mixtures and both radiation types a significant increase in light output at lower temperatures is reported.

In 1999 Buontempo et al. [13] investigated a variety of combinations of four solvents and four solutes. Irradiated with a ${ }^{90} \mathrm{Sr} \beta$-source, when a neutral gas was present, a light yield increase of $\approx 10 \%$ was observed at $-10{ }^{\circ} \mathrm{C}$. Unfortunately again, temperature effects on the PMT were ignored and a setup scheme shows that the cooled and heated cuvette was directly mounted onto the PMT, thus having direct thermal contact.

Finally, the so far last and best documented work was published by Xia et al. [5]. This group built a detector setup much alike the one developed for this work. A ${ }^{137} \mathrm{Cs} \gamma$-ray source was used in a Compton scattering setup that could be heated and cooled. To account for PMT sensitivity changes, an external LED light source was used to generate pulsed reference light, which was led via an optical fibre onto the PMT. With this technique any 'hysteresis' effects were excluded and changes in the PMT sensitivity were measured and corrected. Unfortunately the exact composition of the tested liquids is not reported, and uncertainties for the quantitative results are not presented. With a linear approximation of the shown LAB data, a temperature coefficient of $\approx-0.30 \% /{ }^{\circ} \mathrm{C}$ can be derived from this study.

Even though most of the previous studies have significant conceptual problems or report only coarse results, all show consistently that organic liquid scintillator solutions yield more light at lower temperatures.

This work The influence of temperature changes in the range of -5 to $30^{\circ} \mathrm{C}$ on the light output of LAB based liquid scintillator mixtures are studied, for $\alpha$-particle and electron signals. A small detector setup was developed (Sect. 2) and special care is taken to control eventual changes of the PMT sensitivity due to varying temperature (Sect. 2.1). An energy calibration for all used LAB mixtures has been carried out by irradiating the setup with $\gamma$-ray emitters and fitting the resulting Compton-edge structure in the energy spectrum
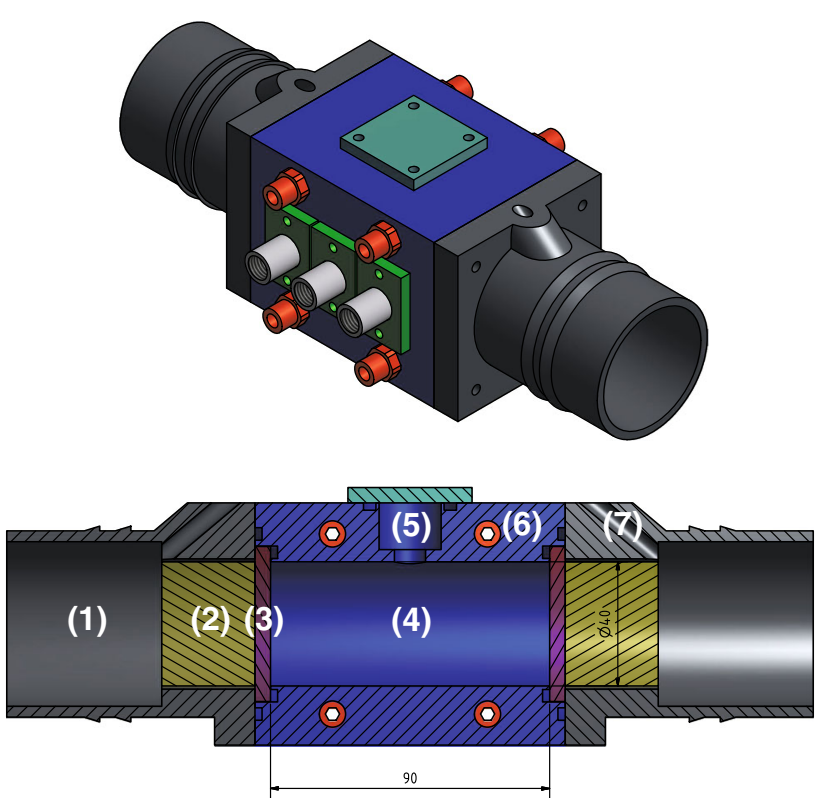

Fig. $13 \mathrm{D}$ (top) and Cutaway view (bottom) of the CAD drawing of the main detector parts with: (1) PMT mounts 3D-printed with ABS (acrylonitrile butadiene styrene) material, (2) UV transparent acrylic light guides, (3) quartz glass windows, $(\mathbf{4})$ cavity of $\approx 110 \mathrm{ml}$ to hold the liquid scintillator, (5) buffer volume \& fill and empty port, (6) four pipes, flushed with glycol-water mixture for cooling and heating, (7) optical fibre feed through to point external LED reference light directly onto the PMT cathodes. The aluminium caps holding the temperature sensors are shown in light grey in the upper drawing

(Sect. 2.2). As this has also been done at multiple temperatures, the changes of the light output for the Compton scattered electrons can be extracted (Sect. 2.3). After evaluating the detector response with an unloaded liquid scintillator mixture $\left(\mathrm{LAB}+5 \mathrm{~g} / \mathrm{l} \mathrm{PPO}+20 \mathrm{mg} / \mathrm{lbis}^{\mathrm{B}} \mathrm{MSB}^{3}\right)$ at different temperatures, measurements with a samarium loaded (Sect. 3.1) and a radon loaded (Sect. 3.2) LAB mixtures were carried out in the given temperature range. Beside the studies of the energy spectra, a pulse shape analysis (Sect. 3.3) yields important insights. All liquid scintillator mixtures were bubbled for 20 min with pure nitrogen before they were filled into the detector, which itself was flushed with nitrogen.

\section{Experimental setup}

The detector itself, shown in Fig. 1, consists of an aluminium block with a cylindrical cavity to hold the liquid scintillator, and two opposing mounted Hamamatsu R2059 PMTs to detect the scintillation light on either side of the vessel. These are thermally decoupled from the liquid by quartz glass windows and $3 \mathrm{~cm}$ long acrylic light guides, since the PMT sensitivity is known to significantly change with tempera-

\footnotetext{
3 1,4-bis(2-methylstyryl)benzol.
} 


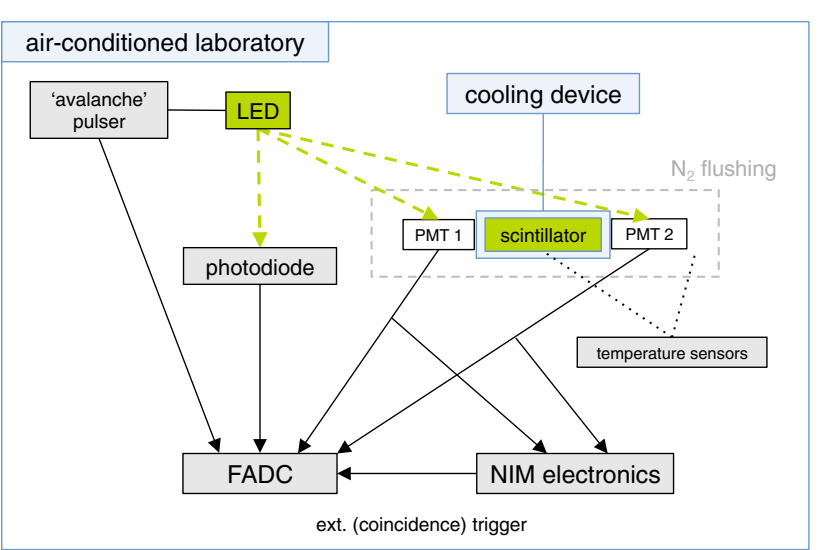

Fig. 2 Schematic drawing of the experimental setup in an airconditioned laboratory with the liquid scintillator detector inside an insulated box with nitrogen atmosphere. All PMT read-out electronics, temperature sensor read-out, as well as the pulser components are outside this box under very stable conditions. Green lines symbolise the light path from the LED light, blue lines indicate cooling and solid black lines the direct read-out by a separate FADC channel

ture $[7,14,15]$. All light guiding parts were optically coupled with silicone grease. The temperature of the active medium is changed by flushing a heated or cooled glycol-water mixture through four pipes in the aluminium block, resulting in temperatures between -5 and $30^{\circ} \mathrm{C}$ inside the liquid scintillator. These temperature changes are detected by two independent temperature sensors (DS18BS20), with an accuracy of 0.5 and $0.044{ }^{\circ} \mathrm{C}$ precision.

As the thermal decoupling of the PMTs cannot be perfect, PMT sensitivity changes are still expected and addressed by a monitoring/correction technique, see Sect. 2.1. To make sure that all PMT gain changes are noticed and can be corrected, an independent light source is used to monitor the PMT response. A schematic overview of the setup and readout electronics is given in Fig. 2.

To achieve PMT coincidence detection and also to include the signals from the LED, the electronic signals from the PMTs are split and simultaneously lead to a NIM ${ }^{4}$ electronics crate for coincidence- and counting logic, and to a $2 \mathrm{GS} / \mathrm{s}$, 10bit FADC ${ }^{5}$ (Acquiris DC-282).

In order to extract a reliable information about the detected amount of light from the recorded pulse traces, the integral of the pulse is used. Furthermore, considering isotropically produced light and homogeneous reflectivity on the vessel walls, the use of the geometric mean of the pulse area values eliminates the dependency on the position of the light generation, resulting in a superior energy resolution for this type of detector. In order for this technique to work, the gain of

\footnotetext{
${ }^{4}$ Nuclear instrumentation module.

5 Flash analog-to-digital converter.
}

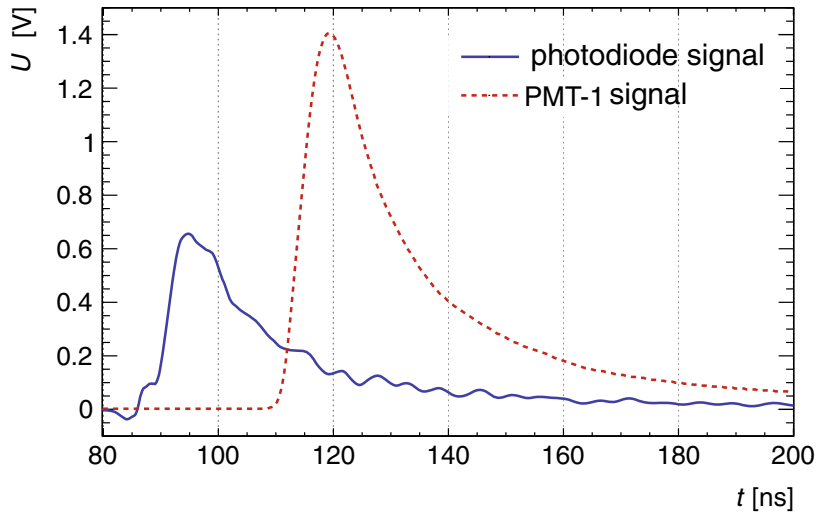

Fig. 3 Comparison of averaged pulse shapes from $\approx 1$ million LED pulser events as they appear on one PMT (red dotted line) and the photodiode (blue solid line). With a rise time shorter than $175 \mathrm{ps}$, the photodiode is able to resolve small 'ripples' of the LED signal, caused by the LED driver. The photodiode signal is linearly amplified

the two PMTs are matched by placing a gamma-ray source at the centre $( \pm 0.5 \mathrm{~mm})$ and adjusting the PMT high voltage.

\subsection{PMT sensitivity control}

With a thermal conductivity of $\lambda \approx 0.2 \mathrm{~W} \mathrm{~m}^{-1} \mathrm{~K}^{-1}$, the acrylic light guides insulate the PMTs well, but not perfectly. Furthermore, the box in which the detector is placed in a nitrogen atmosphere, reaches a thermal equilibrium with the cuvette only after some hours. That is why temperature changes of up to $\approx 10^{\circ} \mathrm{C}$ on the PMTs are expected. The manufacturer for the PMTs used in this setup, states a flat 'temperature coefficient' for the anode sensitivity of $\approx-0.4 \% /{ }^{\circ} \mathrm{C}$ [14], which could be confirmed in this work. As the temperature changes occur on long timescales in an evacuated phototube, they are difficult to quantify for individual and not identical phototubes, and a dedicated control of the PMT sensitivity is necessary.

In order to achieve such a PMT sensitivity control, a custom built pulser drives a LED $(428 \mathrm{~nm})$ with a variable frequency to generate very short light pulses of $\approx 10$ ns (see Fig. 3) that are as similar as possible to the light emitted from the liquid scintillator. This light is then split and lead simultaneously onto the two PMTs and a thermally fully decoupled silicon pin-photodiode (Alphalas UPD-200-PS) outside the $\mathrm{N}_{2}$-box, refer to Fig. 2. This independent signal serves as normalisation and control that the amount of emitted light from the LED is constant.

Proof of concept Extensive tests have been undertaken to show that the light reference by the LED pulser is stable, reliable and that it can be actually used to correct for PMT sensitivity changes by ambient conditions. Long time measurements of up to $24 \mathrm{~h}$ were made to guarantee thermal equi- 


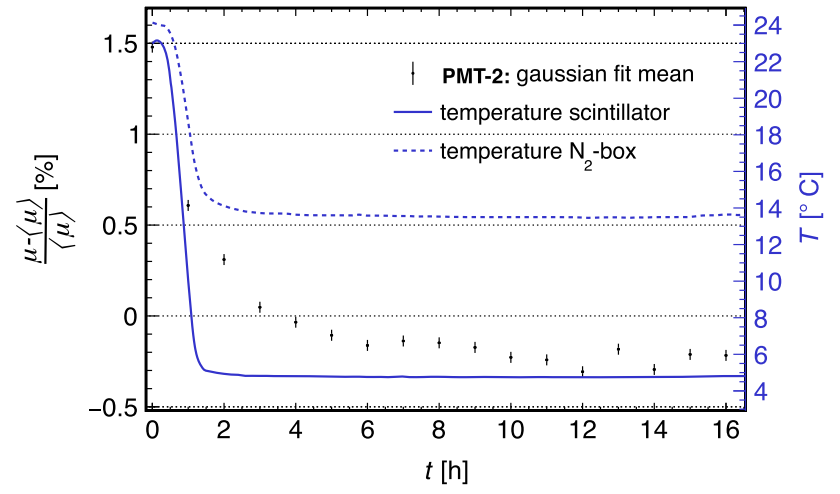

Fig. 4 Time analysis of PMT sensitivity during a temperature change of the liquid scintillator. The solid blue line indicates the temperature (right y-axis) and the black data points are the relative changes of the mean values from a Gaussian fit to the charge spectra, for $1 \mathrm{~h}$ each. The error bars show the $\pm 1 \sigma$ uncertainty from the fit

librium of all parts and gain insights into time stability. These datasets were divided into 1-h- 'blocks' to observe changes on this time base. A pulser rate of $20 \mathrm{~Hz}$ guarantees good statistics without interfering with the signals. As the background rates in the basic LAB solution (LAB $+5 \mathrm{~g} / 1 \mathrm{PPO}+$ $20 \mathrm{mg} / \mathrm{l} \mathrm{bis-MSB}$ ) are low with about $40 \mathrm{~Hz}$ (integral over the full energy spectrum), random coincidences with the pulser are negligible $\sim 10^{-5} \mathrm{~Hz}$, considering a coincidence window of $30 \mathrm{~ns}$. The resulting Gaussian shaped peaks in the charge spectra were fitted with a standard Gaussian and the mean and the fit uncertainty on the mean were extracted. Graphs for this analysis with one PMT and the photodiode are shown in Figs. 4 and 5, respectively. In this case, after $24 \mathrm{~h}$ at room temperature, the cooling device was used to lower the temperature of the liquid scintillator to $5^{\circ} \mathrm{C}$ (left part of the diagrams). After $\approx 4 \mathrm{~h}$, the final temperature is reached, and kept stable by constant cooling. The influence on the PMT sensitivity of the ambient temperature change is clearly visible. After about $5 \mathrm{~h}$, a thermal equilibrium is reached and the differences between the hourly data points turn into a random scatter of the order $\mathscr{O}(0.1-0.2 \%)$. Note that these data points are hour averages and the deviation from the overall mean.

From this observation it was concluded that a time of at least $6 \mathrm{~h}$ needs to be in between the set of a new temperature on the cooling device and actual data taking. In practise, the cool down and warm up periods were during the night and the time span before measuring at least $18 \mathrm{~h}$. Furthermore, it could be shown that the LED pulser and / or the photodiode read-out is long time stable to $\pm 0.2 \%$. These fluctuations are due to fluctuations of the intensity of the LED pulser source. This allows to measure changes in the amount of light hitting the PMTs of the order $\mathscr{O}(1 \%)$.

Finally, the application of a correction to real data, that was taken at different temperatures, needed to be shown. Since

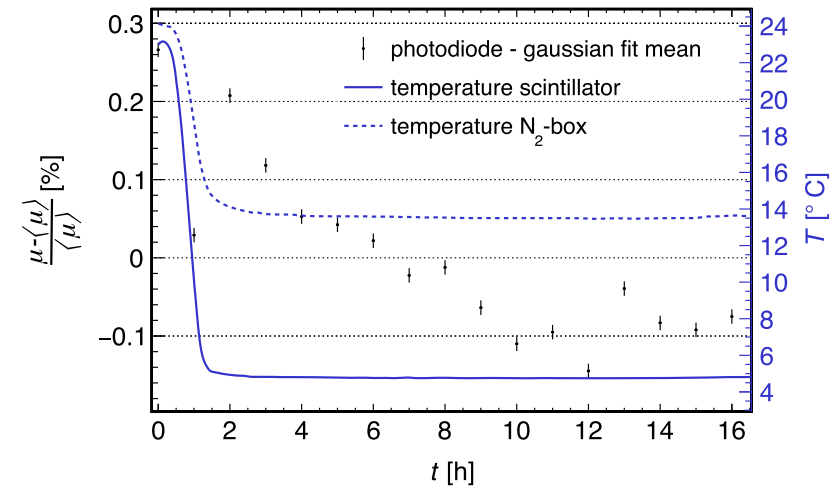

Fig. 5 Time analysis of photodiode signals during a temperature change of the liquid scintillator. The solid blue line indicates the temperature (right y-axis) and the black data points are the relative changes of the mean values from a Gaussian fit to the charge spectra, for $1 \mathrm{~h}$ each. The error bars show the $\pm 1 \sigma$ uncertainty from the fit

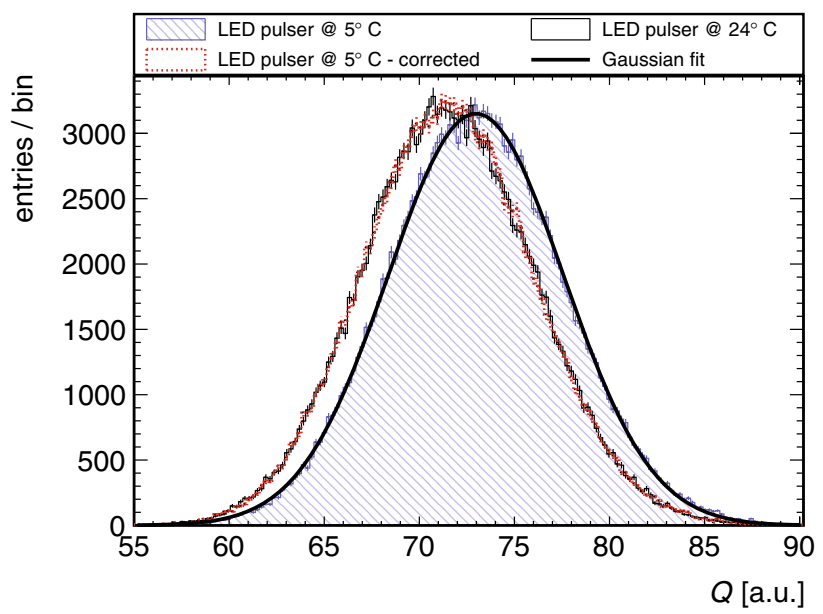

Fig. 6 Comparison of charge spectra from the LED pulser, obtained at $5{ }^{\circ} \mathrm{C}$ (blue) and $24^{\circ} \mathrm{C}$ (black). The red one is generated from the same data as the one in blue, but with the sensitivity correction applied, event by event. The solid black line shows the Gaussian fit to the low temperature data

the pulser trigger signal is also recorded every time the LED is lit, these events can be 'cut' from the data-set and analysed separately. As the area of the pulses are Gaussian distributed around their mean, a standard Gaussian function is used to fit the data and to extract the mean value and fit uncertainty, see Fig. 6. Once this is done for both measurements, the correction factor can be calculated simply as the ratio of the two LED pulser mean values. Since the signal from the photodiode is known to be very stable, any shift from this source can then be subtracted from the correction quotient, as this would indicate a slight change in the light that hits the PMTs. This correction factor can then be used to scale the pulse integral on an event by event basis. As a test, two measurements with a ${ }^{22} \mathrm{Na}$ calibration source were carried out, one at room temperature $(24.20 \pm 0.04)^{\circ} \mathrm{C}$ and one at $(4.80 \pm 0.04)^{\circ} \mathrm{C}$. 


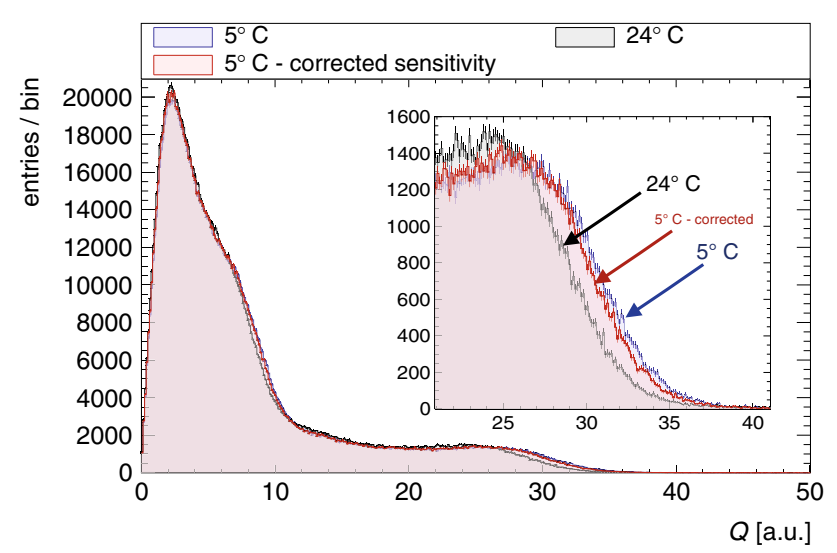

Fig. 7 Comparison of two ${ }^{22} \mathrm{Na}$ charge spectra. The Compton edge at higher energy is clearly visible. The one at the lower energy is less pronounced and merged with the back-scatter feature. In black, the spectrum obtained at $(23.65 \pm 0.04)^{\circ} \mathrm{C}$, and in blue the one from the measurement at $(4.80 \pm 0.04){ }^{\circ} \mathrm{C}$. The red spectrum is generated from the same data as the blue one, but with the sensitivity correction applied, event by event. The inset shows a zoom at the upper Compton edge

A comparison of the spectra from the ${ }^{22} \mathrm{Na}$ calibration source can be seen in Fig. 7. A closer look at Fig. 7 reveals a small change of the Compton edge towards higher charge values for the cooled scintillator. With the application of the gain correction, this effect is reduced and the corrected spectra 'moved' towards the room-temperature position. This is the expected behaviour of the sensitivity correction via a separate light source, as it is applied in this setup.

An important test is the effect of temperature changes on the pulse shapes of the PMTs, in this case illuminated by the LED. While the photodiode is perfectly thermally decoupled from the cooled setup, the PMTs undergo temperature changes. With its $2 \mathrm{GS} / \mathrm{s}$, the FADC has the capability to record the pulse shape of the PMT voltage pulses. Figure 8 shows an overlay of average pulse shapes of LED pulser events on PMT-1, at five temperatures, normalised to the pulse height at room-temperature. The arithmetic average of the voltage value is calculated at each sample position $(0.5$ $\mathrm{ns}$ bin), as well as the variance of the voltage values. Since the variance is very small and the amount of pulses large $(\approx 150 \mathrm{k})$, the uncertainties are too small to be visible here. It can be noted that, clearly the temperature variation has no effect on the shape of the LED pulses on the PMTs, and therefore that the PMTs do not produce different pulse shapes at different temperatures.

\subsection{Energy calibration}

The energy resolution and calibration is obtained by fitting the upper edge of the Compton continuum in the charge spectra of external gamma-ray sources.

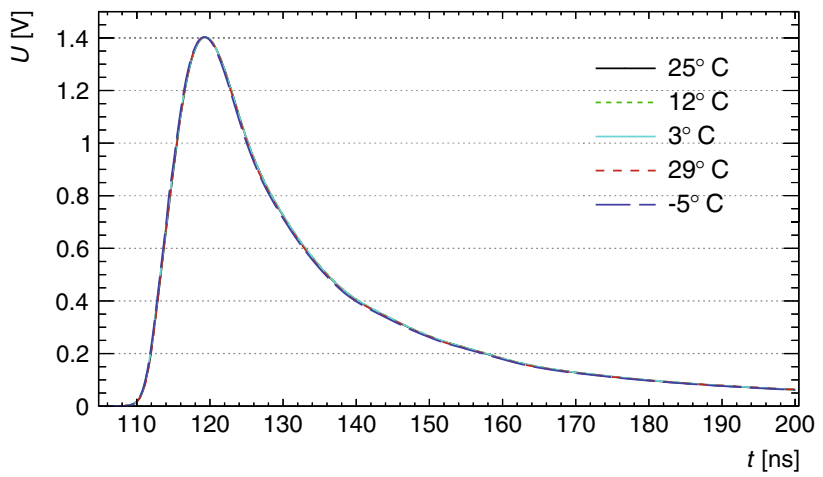

Fig. 8 Average pulse shapes from PMT-1 at five temperatures, generated by the LED pulser and normalised to the pulse height at roomtemperature. The sample by sample average is generated from $\approx 150 \mathrm{k}$ pulses at each temperature. PMT- 2 shows the same behaviour

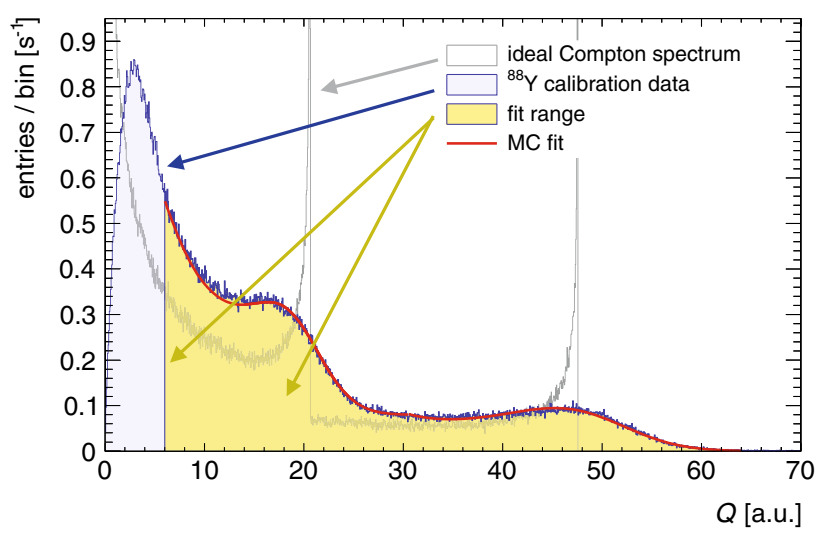

Fig. $9{ }^{88}$ Y calibration spectrum, obtained with $\mathrm{LAB}+5 \mathrm{~g} / 1 \mathrm{PPO}+$ $20 \mathrm{mg} / \mathrm{l}$ bis-MSB. The fit over a wide range is making use of the energy resolution function shown in Fig. 11. The plot shows in blue the actual data, in grey the ideal Compton spectrum and in red the MC simulated and fitted spectrum. The yellow area marks the range of the fit

Firstly, the ideal Compton spectrum is calculated for the individual isotope energies, making use of the analytic crosssection calculation by Klein and Nishina [16]. This ideal spectrum is generated for each individual gamma-line, and those are then combined into a sum-spectrum with the exact branching ratios for the individual decays [17], see the solid black line spectrum in Fig. 9. The used gamma-ray calibration sources $\left({ }^{137} \mathrm{Cs},{ }^{22} \mathrm{Na},{ }^{60} \mathrm{Co},{ }^{207} \mathrm{Bi},{ }^{88} \mathrm{Y}\right)$ cover the energy range from $477 \mathrm{keV}$ to $1612 \mathrm{keV}$ with their respective Compton edge energies.

Secondly, the actually sharp Compton edge is 'smeared' by the energy resolution of the detector and needs to be taken into account for a fit. For every gamma-line, the Compton edge needs to be fitted locally, since the energy resolution is energy dependent itself. Finally, from each edge-fit the position and energy resolution can be extracted. After a rough 'by hand' approximation, the extracted parameters for the position and resolution are used to start a full likelihood fit, using the Minuit routines of the CERN ROOT software packages. 


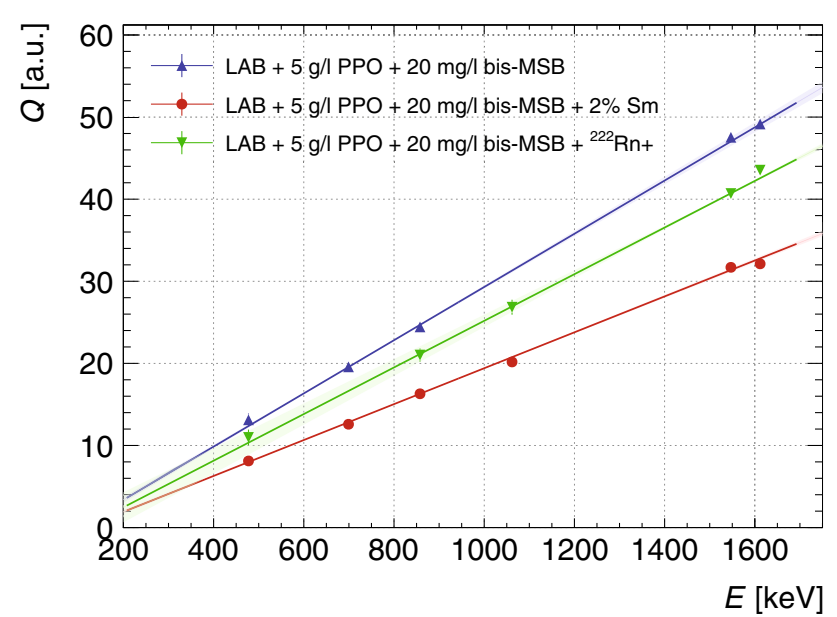

Fig. 10 Energy calibration for three mixtures: 'pure' $\mathrm{LAB}+\mathrm{PPO}+$ bis-MSB (blue), samarium loaded LAB (red) and radon loaded LAB (green), at room-temperature $(24.5 \pm 0.5)^{\circ} \mathrm{C}$. The uncertainties on the individual data points are mostly too small to be visible, as well as the $1 \sigma$ uncertainty band from the linear fit

The ideal spectrum convoluted with an energy dependent energy resolution is drawn with a solid red line in Fig. 9, where the following Eq. 1 is used as analytical function for the energy resolution:

$\frac{\Delta E}{E}=\sqrt{\frac{p_{1}}{E^{2}}+\frac{p_{2}}{E}+p_{3}}$,

with $p_{1}$ as parameter to describe the finite resolution due to electronic noise, $p_{2}$ regarding statistical effects of photon and photo-electron generation and $p_{3}$ the influence of the position of the light generation and attenuation. For all three LAB mixtures used in this work, the energy calibration and energy dependent energy resolution obtained from these fits are shown in Figs. 10 and 11, respectively. A detailed description of the used loading technique and properties of these mixtures can be found at the beginning of the Sects. 3.1 and 3.2 .

As expected, the LAB mixture with metal loading emits much less light (see Fig. 10), or more precisely, much less light is detected, due to reduced transparency of the scintillator. This effect can also be seen in UV-transmission measurements. It is also the main reason why the energy resolution is worse for samarium loaded LAB. Another factor increasing mainly the uncertainty of the energy resolution for lower energies in this scintillator mixture, is the alpha peak from the ${ }^{147} \mathrm{Sm}$, disturbing the Compton edge fitting.

Moreover it can be seen that the energy resolution in the 'pure' scintillator mixture $(\mathrm{LAB}+5 \mathrm{~g} / \mathrm{lPO}+20 \mathrm{mg} / \mathrm{l}$ bisMSB) is not affected when ${ }^{222} \mathrm{Rn}$ is added to the liquid only by diffusion, see green and blue data in Fig. 11. Details of the loading process will be discussed in Sect. 3.2. The reason why the green data points are below the blue ones in Fig. 10 is

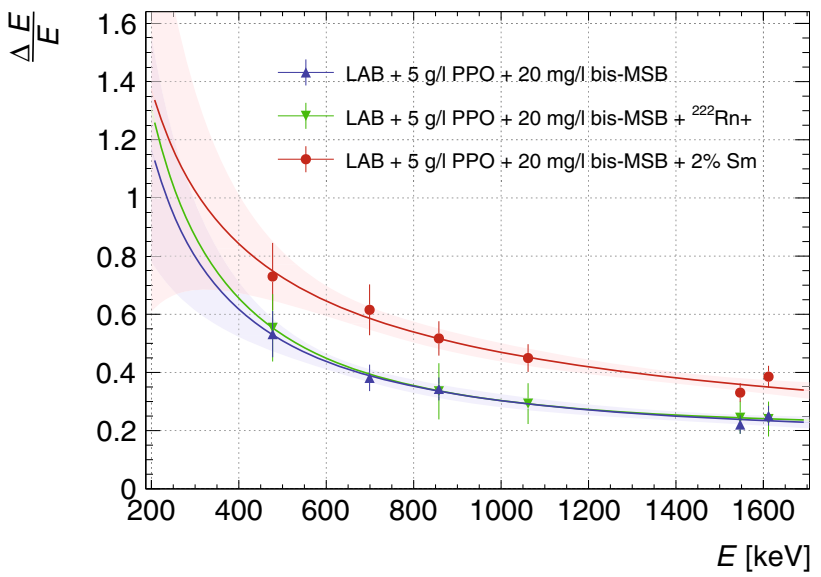

Fig. 11 Energy dependent energy resolution for three mixtures: 'pure' $\mathrm{LAB}+\mathrm{PPO}+$ bis-MSB (blue), samarium loaded LAB (red) and radon loaded LAB (green), at room-temperature $(24.5 \pm 0.5){ }^{\circ} \mathrm{C}$. The fit function (see Eq. 1) is shown with its $1 \sigma$ uncertainty band, obtained from the fit

the fact that for the radon loaded LAB measurement a lower high voltage on the PMTs had to be used, since they were different PMTs (but same type) and the activity of the radon was high.

\subsection{Light yield calibration at different temperatures}

Previous studies by Homma et al. [10-12], Buontempo et al. [13] and especially Xia et al. [5] have shown or at least indicated that an increase in electron light yield with lower temperatures has to be expected. Thus, the calibration technique described in the previous section is applied at different temperatures. This is especially important to identify a possible difference in the temperature quenching between $\alpha$-particles and electrons.

Four energies from three different gamma-ray emitting isotopes $\left({ }^{22} \mathrm{Na},{ }^{207} \mathrm{Bi}\right.$ and ${ }^{137} \mathrm{Cs}$ ) were used to determine the position of their Compton edges at five temperatures (see Fig. 12) in the 'pure' scintillator mixture ( $\mathrm{LAB}+5 \mathrm{~g} / 1 \mathrm{PPO}+$ $20 \mathrm{mg} / \mathrm{l}$ bis-MSB). Combining the individual results for each energy, Fig. 12 shows the change in charge versus temperature. The slope of the linear fit is $(-0.173 \pm 0.018) \% /{ }^{\circ} \mathrm{C}$, indicating a change in light yield in the same order of magnitude as the results reported in [5]. There, only diagrams with data points and splines are presented and no linear approximation is performed. Therefore only a very rough 'by hand' estimate of $\approx-0.32 \% /{ }^{\circ} \mathrm{C}$ can be used for comparison with the data obtained in this work.

A comparison of the linear fit slopes for the individual isotopes and their energies will be presented in final discussion of all results in Sect. 4. 


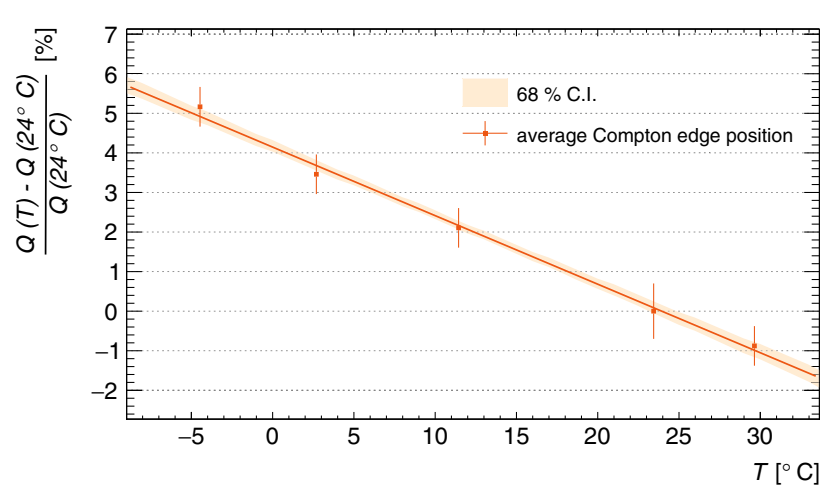

Fig. 12 Charge (light yield) deviation of fitted Compton edges at five different temperatures, in 'pure' $5 \mathrm{~g} / \mathrm{lPPO}+20 \mathrm{mg} / \mathrm{l}$ bis-MSB. Each data point contains the combined data for four energies and is normalised to the room-temperature value $(23.44 \pm 0.04){ }^{\circ} \mathrm{C}$. Uncertainties consist of the variance between the values from the different energies and the fit uncertainty. The light orange band shows the $68 \%$ confidence belt, taken from the linear fit (solid orange line)

\section{Measurements and analysis}

\subsection{Samarium loaded LAB}

At the Brookhaven National Laboratory (BNL), natural samarium was loaded into LAB based liquid scintillators, using the surfactant $\mathrm{TMHA}^{6}$ in a single-stage solventsolvent extraction procedure, which can be used to form organometallic compounds that can be dissolved in an organic liquid.

Natural samarium contains $(15.01 \pm 0.03) \%$ of the isotope ${ }^{147} \mathrm{Sm}$, which is $\alpha$-instable with a half-life of $T_{1 / 2}=(1.070 \pm$ $0.009) \times 10^{11} \mathrm{y}$ and $E_{\alpha}=2.248 \mathrm{MeV}[18,19]$. The mixture used for the measurements in this work contained about $2 \%$ natural samarium, resulting in a 'constant' activity of about $200 \mathrm{~Bq}$ in the $100 \mathrm{ml}$ volume of the detector used, from this decay. Although natural samarium also contains $11.24 \%$ of the $\alpha$-instable isotope ${ }^{148} \mathrm{Sm}$, with its half-life of $T_{1 / 2}=$ $7 \times 10^{15} \mathrm{y}$ [17], its activity of $\approx 2 \times 10^{-3} \mathrm{~Bq}$ is negligible in this case.

Analysis Figure 13 shows a calibrated light yield spectrum at room temperature. The fit function is a Gaussian, though as the samarium peak showed a high energy tail, other shapes were tried too (Landau- and skewed normal distribution), neither did fit well nor improved the goodness of fit significantly. Thus the model independent, conservative Gaussian shape was chosen, especially as no physics motivation for any other shape was found. The overall activity in the setup was too low for pile-up events, and no database entry of any $\mathrm{X}$-ray emission for ${ }^{147} \mathrm{Sm}$ was found.

\footnotetext{
6 3,5,5-trimethyl-hexenoic acid.
}

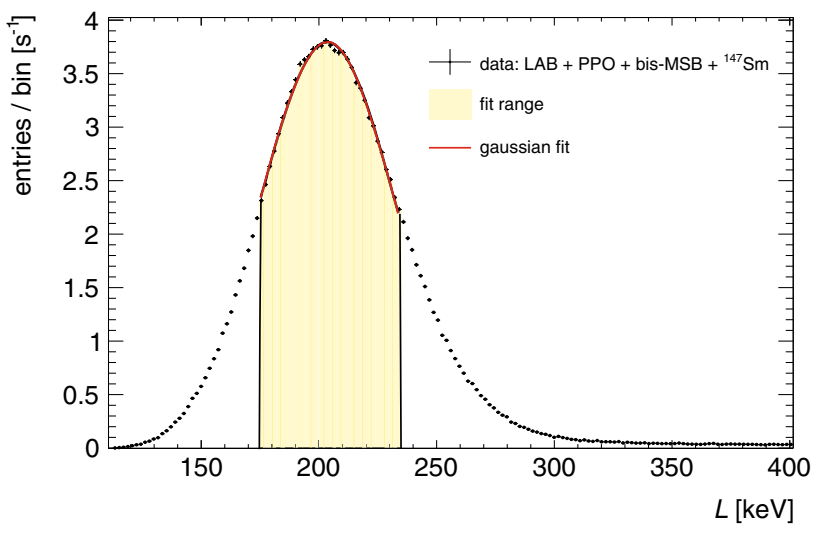

Fig. 13 Light yield spectrum at room-temperature $\left(24.62 \pm 0.04{ }^{\circ} \mathrm{C}\right)$ of $\mathrm{LAB}+5 \mathrm{~g} / 1 \mathrm{PPO}+20 \mathrm{mg} / 1$ bis-MSB $+2 \%{ }^{\text {nat }} \mathrm{Sm}$. The yellow shaded area marks the $1 \sigma$ fit range for the Gaussian fit

Table 1 List of uncertainties considered in the analysis of the ${ }^{\text {nat }} \mathrm{Sm}$ loaded LAB mixture at room-temperature $\left(24.62 \pm 0.04^{\circ} \mathrm{C}\right)$

\begin{tabular}{ll}
\hline Uncertainty & Value (\%) \\
\hline Fit & 0.08 \\
Fit range & 0.47 \\
Pulser stability & 0.35 \\
Variance between rep. measurements & 0.2 \\
Sum & $\mathbf{1 . 1}$ \\
\hline
\end{tabular}

Therefore, for all ${ }^{\text {nat }} \mathrm{Sm}-\mathrm{LAB}$ temperatures the following fit routine was applied: First a simple Gaussian fit with a wide range is performed, to roughly identify the peak position and width. In a second step, the fit range is confined to $1 \sigma$ around the mean of the first fit. The second (actual) fit is in this case restricted to $1 \sigma$ to avoid any distortion due to the high energy tail. This is incorporated in the final analysis and evaluation by a 'fit range uncertainty', which is calculated as the variance of the fit mean values of Gaussian approximations with $1 \sigma$, $2 \sigma$ and $3 \sigma$ fit range. For the room-temperature measurements a list of the individual uncertainties considered is given in Table 1.

All temperature varied measurements have been performed at least twice, except the one for $30^{\circ} \mathrm{C}$ which has only been done once. The sequence of the measurement was chosen for practical reasons to $24^{\circ} \mathrm{C} \rightarrow 5^{\circ} \mathrm{C} \rightarrow 14^{\circ} \mathrm{C} \rightarrow$ $24^{\circ} \mathrm{C} \rightarrow-4^{\circ} \mathrm{C} \rightarrow 24^{\circ} \mathrm{C} \rightarrow 30^{\circ} \mathrm{C}$ and then repeated without the two $24^{\circ} \mathrm{C}$ steps in between. As this does not allow to check for any systematic effects of the $T$ changes in the detector system (e.g. hysteresis effects), a more systematic approach for the temperature sequence was chosen in the radon loaded LAB studies, presented in the next Sect. 3.2.

As room-temperature is an arbitrary but natural scale for liquid scintillator measurements, all changes observed with temperature are normalised to the room-temperature value. 


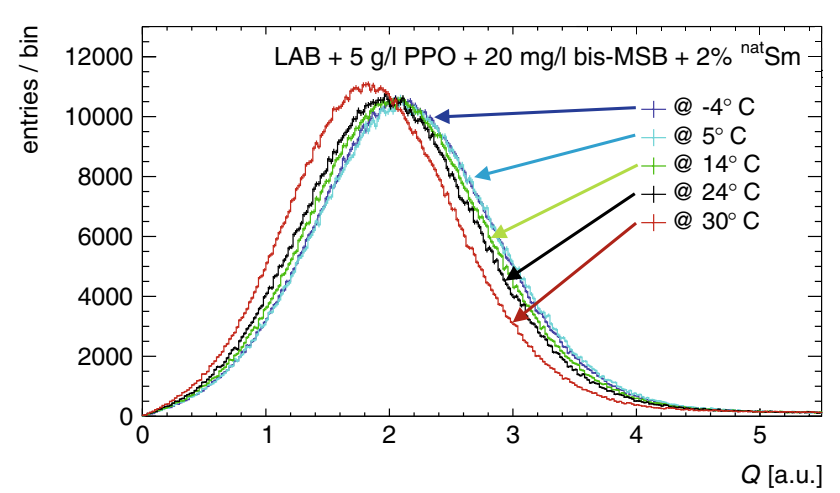

Fig. 14 Comparison of ${ }^{\text {nat }} \mathrm{Sm}$ loaded LAB charge spectra at different temperatures, with the PMT sensitivity correction already applied

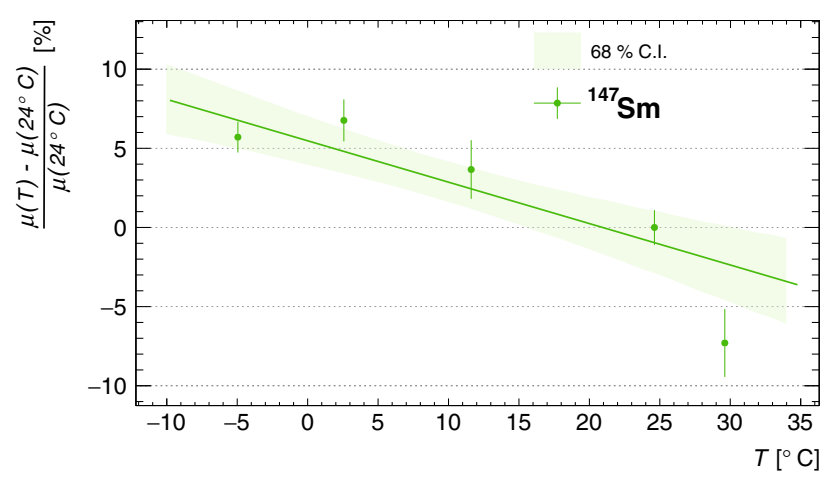

Fig. 15 Shift of the Gaussian fit mean values versus liquid scintillator temperature, for the ${ }^{\text {nat }} \mathrm{Sm}$ loaded $\mathrm{LAB}$ measurements. Each data point contains the combined values for the repeated measurements and is normalisation to the room-temperature value, $(24.62 \pm 0.04)^{\circ} \mathrm{C}$. The green shaded area marks the $1 \sigma$ confidence band obtained from the linear fit

Figure 14 gives an impression of how the temperature change affects the ${ }^{147} \mathrm{Sm}$ peak position, after the PMT sensitivity correction is applied to correct for any ambient temperature effects on the PMTs.

In Fig. 14 the systematic shift towards a higher light yield with decreasing temperature is clearly visible and a linear fit yields a slope of $(-0.26 \pm 0.02) \% /{ }^{\circ} \mathrm{C}$. The data for the $30^{\circ} \mathrm{C}$ measurement seems to be shifted disproportionately towards lower charge values. The visual effect is increased by the observation that the peak is also more narrow. Also in the final analysis of the peak shift, the $30^{\circ} \mathrm{C}$ data seems to deviate of the seemingly linear trend of the other data points, see Fig. 15.

In order to incorporate also the results from the radon loaded LAB measurements and pulse shape analysis, a full discussion of the results is given in Sect. 4.

\subsection{Radon loaded LAB}

In order to extend the study of the light yield temperature behaviour with other $\alpha$-emitting isotopes, preferably
Table 2 Overview table for the relevant part of the ${ }^{222} \mathrm{Rn}$ decay chain. All decays have a branching ratio of at least $99.98 \%$ [17]

\begin{tabular}{lll}
\hline Decay & $\mathrm{T}_{1 / 2}$ & $E_{\alpha / \beta}(\mathrm{MeV})$ \\
\hline${ }^{222} \mathrm{Rn} \rightarrow{ }^{218} \mathrm{Po}$ & $3.82 \mathrm{~d}$ & 5.49 \\
${ }^{218} \mathrm{Po} \rightarrow{ }^{214} \mathrm{~Pb}$ & $3.10 \mathrm{~min}$ & 6.00 \\
${ }^{214} \mathrm{~Pb} \rightarrow{ }^{214} \mathrm{Bi}$ & $26.8 \mathrm{~min}$ & 1.0 \\
${ }^{214} \mathrm{Bi} \rightarrow{ }^{214} \mathrm{Po}$ & $19.9 \mathrm{~min}$ & 3.3 \\
${ }^{214} \mathrm{Po} \rightarrow{ }^{210} \mathrm{~Pb}$ & $164.3 \mu \mathrm{s}$ & 7.69 \\
\hline
\end{tabular}

at higher energies and with another loading technique, the decay chain of ${ }^{222} \mathrm{Rn}$ was chosen. This isotope can be obtained from ${ }^{226} \mathrm{Ra}$, which is an $100 \% \alpha$-emitter with a halflife of $T_{1 / 2}=1600 \mathrm{y}$, decaying solely to ${ }^{222} \mathrm{Rn}$. As radon is a gas, the isotope escapes the porous ceramic Radium source and can be transferred via gas exchange. This radon gas is used in a nitrogen atmosphere to 'bubble' through a LAB mixture. For better comparison, the same 'pure' LAB + $5 \mathrm{~g} / \mathrm{l} \mathrm{PPO}+20 \mathrm{mg} / \mathrm{l}$ bis-MSB solution (without samarium) was used. After circulating the nitrogen - radon atmosphere through the LAB mixture for about $72 \mathrm{~h}$, an activity of about $200 \mathrm{kBq}$ was introduced into the liquid scintillator. This high activity was beyond expectation and the setup had to rest for several weeks to reduce the activity to about $1 \mathrm{kBq}$.

Even though the ${ }^{222} \mathrm{Rn}$ decay chain ends only at the stable ${ }^{206} \mathrm{~Pb}$, in the scope of this work already the isotope ${ }^{210} \mathrm{~Pb}$ presents the end of the chain with a half-life of $T_{1 / 2}=22.2 y$, leading to an activity too low to be visible. This short chain produces three $\alpha$-particles and has two $\beta^{-}$-decays, refer to Table 2.

The first $\alpha$-decay of ${ }^{222} \mathrm{Rn}$ has the longest half-life of $T_{1 / 2}=3.82 \mathrm{~d}$ and thus, as the other isotopes decay with minutes and micro-seconds, the chain is very fast at secular equilibrium. The first $\alpha$-energy of $5.49 \mathrm{MeV}$ is close to the one from the following ${ }^{218} \mathrm{Po}$ with $6.00 \mathrm{MeV}$, and can therefore not be distinguished with this detector setup. Nevertheless, in a combined fit, it is possible to extract the individual light yields. The resulting ${ }^{214} \mathrm{~Pb}$ decays via $\beta^{-}$-emission to ${ }^{214} \mathrm{Bi}$, with an end-point energy of $1.0 \mathrm{MeV}$. Subsequently ${ }^{214} \mathrm{Bi}$ changes to ${ }^{214} \mathrm{Po}$, emitting an electron with the maximum energy of 3.3 MeV. Its very short half-life of $T_{1 / 2}=164.3 \mu \mathrm{s}$ proves useful for tagging this and the following $\alpha$-decay to the final ${ }^{210} \mathrm{~Pb}$. This last $\alpha$-decay has an energy of $7.69 \mathrm{MeV}$, which separates it well from the preceding $\alpha$-particles.

This LAB mixture with dissolved radon was used to observe light output changes at five temperatures, in the following sequence: $24^{\circ} \mathrm{C} \rightarrow 10^{\circ} \mathrm{C} \rightarrow 5^{\circ} \mathrm{C} \rightarrow-4^{\circ} \mathrm{C} \rightarrow 5^{\circ} \mathrm{C}$ $\rightarrow 10^{\circ} \mathrm{C} \rightarrow 30^{\circ} \mathrm{C} \rightarrow 24^{\circ} \mathrm{C} \rightarrow 10^{\circ} \mathrm{C} \rightarrow 5^{\circ} \mathrm{C}$. No systematic effect has been observed, repeated measurements scattered around their mean randomly, without a sign preference. A calibrated light yield spectrum is shown in Fig. 16. 


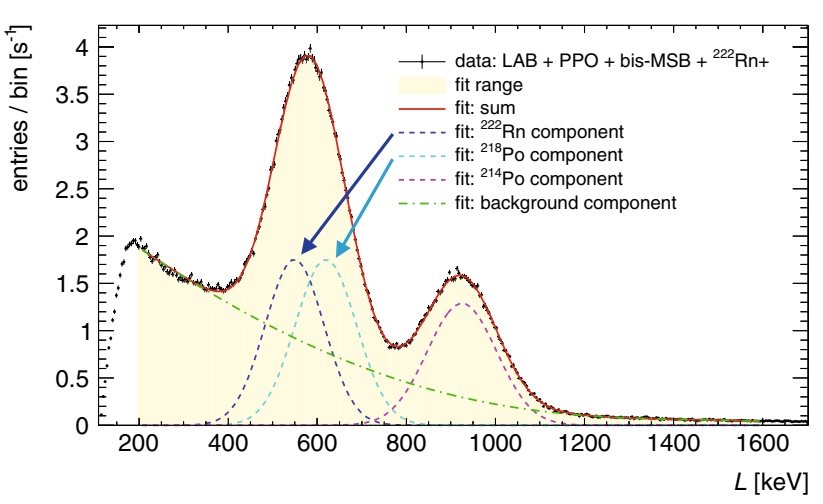

Fig. 16 Calibrated light output of radon loaded LAB $(+5 \mathrm{~g} / \mathrm{l}$ PPO + $20 \mathrm{mg} / \mathrm{l}$ bis-MSB) at room-temperature. The fit function (red line) is the sum of three Gaussian functions (dashed blue, cyan and pink lines) and a $6^{\text {th }}$ order polynomial (dashed green line). The fit range is $200-1600$ $\mathrm{keV}$

Analysis As can be seen in Fig. 16, the two $\beta$-decays present a significant background for the quenched $\alpha$-peaks in the energy spectrum. Different strategies can be applied to still extract the relevant peak shifts with varying temperature. One option is to analyse the energy spectrum as it is and either subtract the $\beta$-background via fitting it first, or include it in a combined fit with the $\alpha$-peaks. Another option is to make use of the short $164.3 \mu$ s half-life of ${ }^{214} \mathrm{Po}$ and apply a timecoincidence cut to separate ${ }^{214} \mathrm{Bi}$ - ${ }^{214} \mathrm{Po}$ (Bi-Po coincidence) event pairs from the decay chain and thus the energy spectra. Even though this second option seems very promising, the first one yields smaller uncertainties and will be presented here. The second analysis can be found in the Appendix A and confirms the absolute numbers of the 'combined fit method' nicely.

In a previous study by Krosigk et al. [20], a $11 \mathrm{LAB}+2$ g/l PPO sample was analysed, which had been placed inside the water-filled SNO detector in 2008. The liquid scintillator was also 'contaminated' with dissolved radon gas and the energy spectrum shows very similar features as are visible in Fig. 16. The background, mainly consisting of the two $\beta$ decays, was approximated with a 6th order polynomial and then subtracted. Krosigk et al. excluded the $\alpha$-peak region from their background fit.

In the analysis of this work, the $\alpha$-peaks and the $\beta$ backgrounds were fitted in one combined fit, visible in Fig. 16. The fit function is the sum of three Gaussian functions and a 6th order polynomial. In order for this fit to properly converge, some of the 15 parameters needed to be constrained, mainly those of the two close $\alpha$-peaks. As they stem from subsequent decays with no other branches, they have the same activity. The strength parameter was therefore the same for both Gaussians, yet it could float freely. Also the meanparameters of the first two Gaussians are constrained, such that they can not take the same value. The mean-parameter
Table 3 Gaussian fit mean values for $\alpha$-particles in the ${ }^{222} \mathrm{Rn}$ decay chain in $\mathrm{LAB}$, at room-temperature

\begin{tabular}{llll}
\hline$\alpha$-Emitter & $E_{\alpha}(\mathrm{MeV})$ & $L(\mathrm{MeV})[20]$ & $L(\mathrm{MeV})$ (this work) \\
\hline${ }^{222} \mathrm{Rn}$ & 5.49 & $0.56 \pm 0.02$ & $0.55 \pm 0.02$ \\
${ }^{218} \mathrm{Po}$ & 6.00 & $0.64 \pm 0.02$ & $0.62 \pm 0.02$ \\
${ }^{214} \mathrm{Po}$ & 7.69 & $0.92 \pm 0.02$ & $0.93 \pm 0.02$ \\
\hline
\end{tabular}

The light output is given in electron equivalent energy, after calibration. Uncertainties stem from light yield calibration and the fit

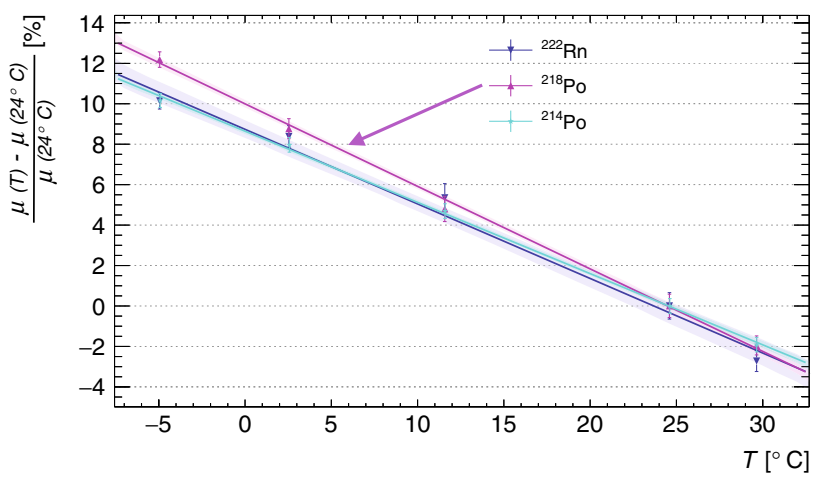

Fig. 17 Shift of the $\alpha$-peak mean values (from the combined fit in Fig. 16) vs. liquid scintillator temperature. Each temperature point is the average of the repeated individual measurements and these are normalised to the average room-temperature value at $(24.5 \pm 0.04){ }^{\circ} \mathrm{C}$. The shaded areas depict the $1 \sigma$ confidence band, obtained from the linear fit

Table 4 Temperature coefficients (slope values) and uncertainties for the three $\alpha$-isotopes in the radon loaded LAB mixture $(+5 \mathrm{~g} / 1 \mathrm{PPO}+$ 20mg/l bis-MSB), obtained via the linear fits in Fig. 17

\begin{tabular}{ll}
\hline Isotope & Temperature coefficient $\left[\% /{ }^{\circ} \mathrm{C}\right]$ \\
\hline${ }^{222} \mathrm{Rn}$ & $-0.37 \pm 0.02$ \\
${ }^{218} \mathrm{Po}$ & $-0.41 \pm 0.02$ \\
${ }^{214} \mathrm{Po}$ & $-0.35 \pm 0.02$ \\
\hline
\end{tabular}

of the first Gaussian has to be in a range below their sumGaussian peak mean and vice versa. With these rough constraints, the fits proved to be very stable and well converging throughout the analysis, also for different temperatures. The room-temperature results for the peak positions are listed in Table 3 and are well compatible with the values obtained by Krosigk et al.

When analysing the full energy spectrum with the described fit at different temperatures, changes of the individual $\alpha$-peak positions can be extracted. The resulting slopes are shown in Fig. 17 and the values are listed in Table 4.

The repeated temperatures vary less than $0.5^{\circ} \mathrm{C}$, and for the final analysis their average has been used. Of course, also the PMT sensitivity correction is applied before analysing the spectra. 


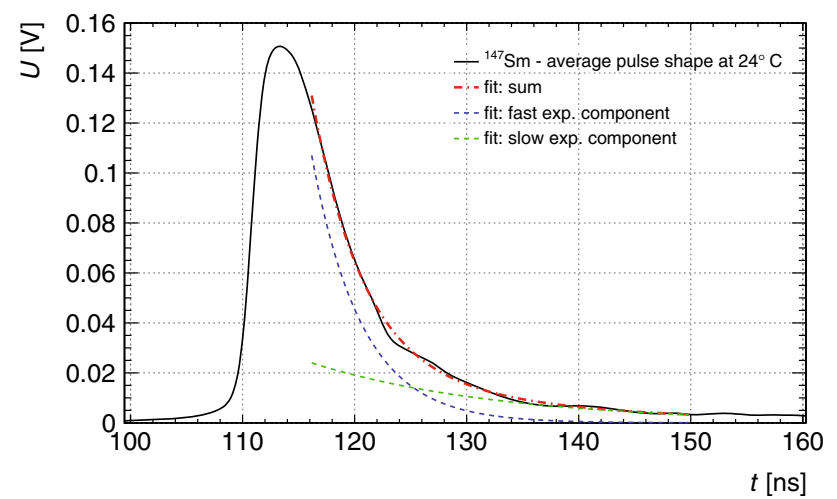

Fig. 18 Exponential fit to an averaged pulse shape from ${ }^{147} \mathrm{Sm} \alpha$-peak, acquired at $(24.62 \pm 0.04)^{\circ} \mathrm{C}$ and calculated from $\sim 1 \mathrm{M}$ pulses. The red dashed line shows the actual fit function, whereas the blue and green dotted lines depict the fast and slow exponential component (see Eq. 2), respectively

\subsection{Pulse shape analysis}

Since the Acquiris FADC is able to take a voltage sample from the PMTs every $0.5 \mathrm{~ns}$, it is possible to perform a pulse shape analysis (PSA) with this experimental setup. As the variations between individual pulse shapes are very large, averaged pulses will be used for comparisons and fits. For a given pulse ensemble, at each sample point, the arithmetic average of the voltage values is calculated, as well as the variance between these pulses. With a typical size of one million pulses, the uncertainty (variances) are very small, and mostly not visible in the following graphs.

To illustrate the analysis principle Fig. 18 shows an averaged pulse shape from the ${ }^{147} \mathrm{Sm} \alpha$-peak obtained from the samarium loaded LAB mixture, at room-temperature. The typical, very fast rise to the maximum is clearly visible, as well as a slower falling slope, followed by an even less steep slope, which can be attributed to a fast and slow decay component. The slight kink visible at $\approx 123 \mathrm{~ns}$ might be attributed to a change in decay constants. Although it was demonstrated that it is not originating from any PMT effects (see PMT pulse shapes in Sect. 2.1, Fig. 8), these deviations from a perfectly smooth curve can not be fully explained.

In order to quantify the changes in the slopes, the following composition of two exponential functions was chosen, convoluted with a time response function with a Gaussian resolution and fitted to the average pulse shapes:

$U(t)=\left(e^{c_{1}-t / \tau_{\text {fast }}}+e^{c_{2}-t / \tau_{\text {slow }}}\right) \otimes \operatorname{gaus}(\sigma)$

with $U$ - voltage, $t$ - time, $\tau_{\text {fast }}, \tau_{\text {slow }}$ - decay constants, $c_{1}, c_{2}$ - constants and $\sigma$-resolution of the time response function. It was found that a two component exponential function fitted the data best, all attempts with a third component lead to fits with a vanishing third part. Also the rising edge was not

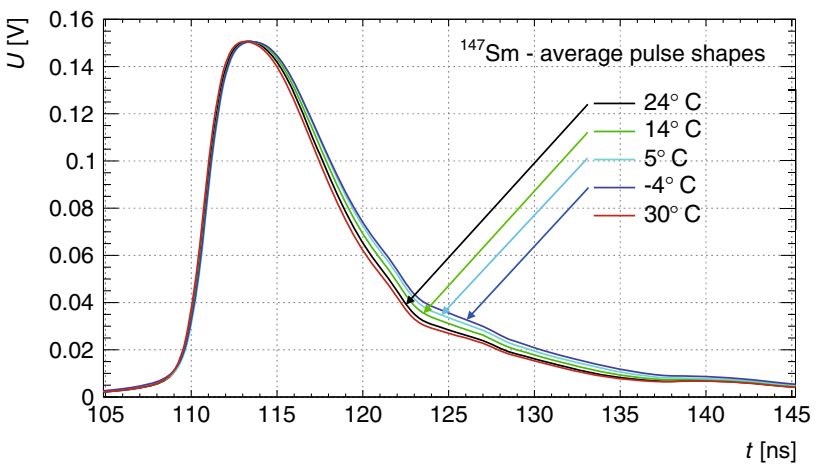

Fig. 19 Average pulse shapes from ${ }^{147} \operatorname{Sm} \alpha$-peak, acquired at different temperatures. Each line is calculated from $\sim 1 \mathrm{M}$ pulses, and normalised to the height of the room-temperature shape

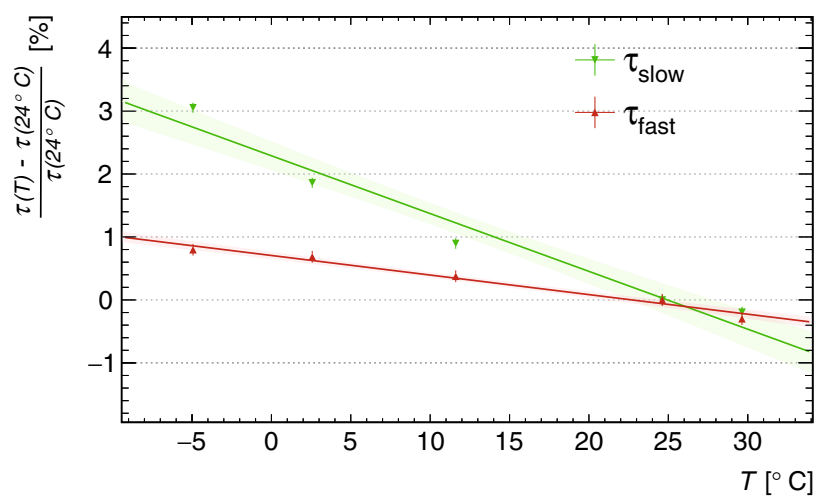

Fig. 20 Change of the decay constant fit values of averaged pulse shapes from ${ }^{147} \mathrm{Sm}$, at different temperatures. The slope of the linear fit for the slow component is $(-0.09 \pm 0.003) \% /{ }^{\circ} \mathrm{C}$. The shaded areas show the $68 \%$ confidence band of the fit

included here, since it is the same for all temperatures (see e.g. Fig. 19). Once $c_{1}$ and $c_{2}$ are fixed to their fit value at roomtemperature, the approximations for the other temperatures reveal the change in the decay constants. These are e.g. for ${ }^{147} \mathrm{Sm}$ at room-temperature:

$\tau_{\text {fast }}=(4.48 \pm 0.01) \mathrm{ns} \quad$ and $\quad \tau_{\text {slow }}=(16.90 \pm 0.01) \mathrm{ns}$

for the fast and slow component, respectively.

Samarium loaded $L A B$ Figure 19 shows a comparison of averaged pulse shapes from the ${ }^{147} \mathrm{Sm} \alpha$-peak, at different temperatures.

It is clearly visible that the shapes change between $115 \mathrm{~ns}$ and $140 \mathrm{~ns}$. With lower temperatures, the falling slope of the pulses becomes less steep. In contrast, the rising edge is the same for all temperatures. The changes that these constants undergo is illustrated in Fig. 20. It is quite clear that the second, slow component is stronger expressed with lower temperatures, showing an increase of about $4 \%$ over the range of $35^{\circ} \mathrm{C}$. 


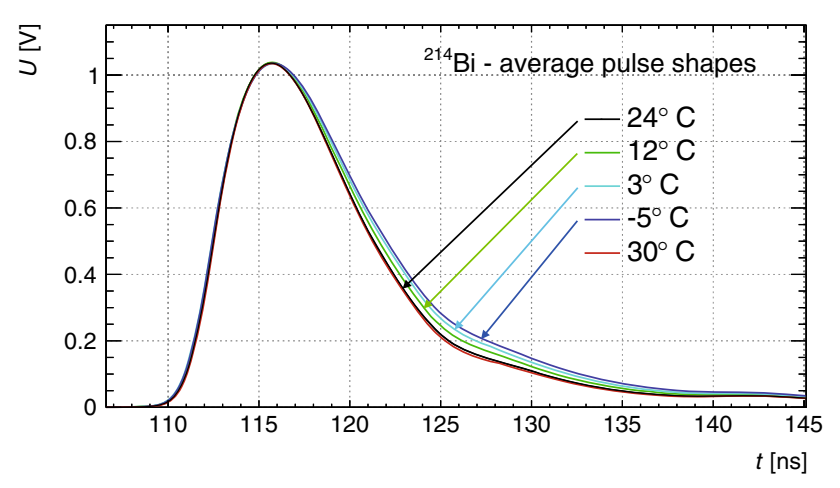

Fig. 21 Average pulse shapes from ${ }^{214} \mathrm{Bi} \beta$-decays in radon loaded $\mathrm{LAB}$, at different temperatures. Each line is calculated from $\sim 1 \mathrm{M}$ pulses, and normalised to the height of the room-temperature shape

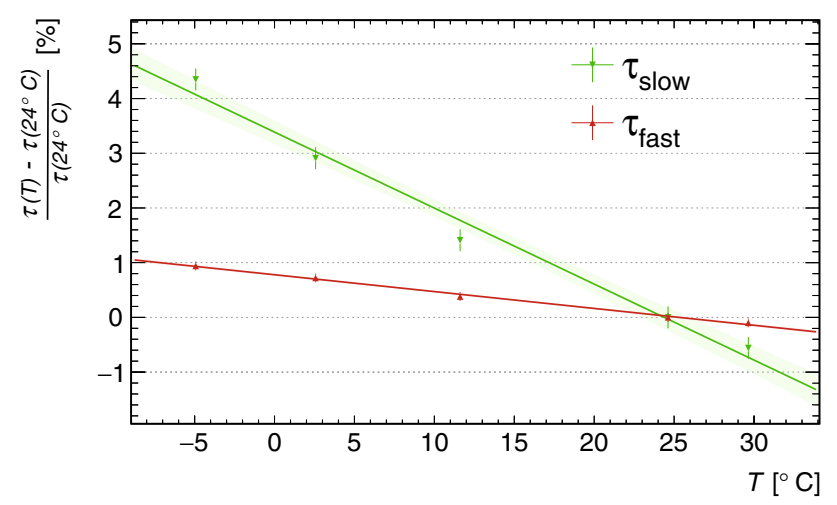

Fig. 22 Change of the decay constant fit values of averaged pulse shapes from ${ }^{214} \mathrm{Bi}$, at different temperatures. The slope of the linear fit for the slow component is $(-0.14 \pm 0.007) \% /{ }^{\circ} \mathrm{C}$. The shaded areas show the $1 \sigma$ confidence band from the fit

Radon loaded $L A B$ For the PSA of the radon loaded LAB measurements, it is sensible to use the data-sets with the isolated $\beta$ - and $\alpha$-events. This is done by applying the same time and energy cuts as in the alternative analysis of the peak shifts, described in the Appendix A.

When analysing the averaged pulse shapes from the ${ }^{214} \mathrm{Bi}$ $\beta$-decays, they seemingly resemble the prolonged pulses that can be seen from the $\alpha$ activity in samarium loaded LAB (Fig. 19). Here again, with lower temperatures the pulse get longer, while with rising temperature the slow decay component is to be suppressed (Fig. 21). To quantify this behaviour and compare with the results from the $\alpha$-decays, the same fit as for the samarium loaded LAB is applied (Fig. 18, Eq. 2). The results for the two decay constants from the fitting are shown in Fig. 22.

As it has been observed for the $\alpha$-decay of ${ }^{147} \mathrm{Sm}$, the fast decay component experiences only minor changes with temperature variation of about $\approx 1 \%$ over a range of $35^{\circ} \mathrm{C}$. On the other hand, even though the uncertainties are larger, the slow decay part changes $\approx 5 \%$ over the same range. This is significantly more and close to the observation from ${ }^{147} \mathrm{Sm}$, even though here it is electrons instead of $\alpha$-particles.

This similar behaviour can again be confirmed with yet another $\alpha$-source, the well isolated ${ }^{214} \mathrm{Po}$ decay in the radon loaded LAB. Again, applying the energy- and time-cuts described in the Appendix A ensures the very good separation of the polonium decay from unwanted backgrounds. Again, while the fast decay component exhibits only a small change with temperature, the slow decay becomes $\approx 4 \%$ stronger at $35^{\circ} \mathrm{C}$ lower temperature. This is very similar to changes observed from ${ }^{147} \mathrm{Sm}$, and less than that of the ${ }^{214} \mathrm{Bi}$ $\beta$-decays (see Fig. 22).

\section{Discussion and conclusions}

If the results from all energies are combined and a linear approximation is assumed, a temperature coefficient of

$(-0.17 \pm 0.02) \% /{ }^{\circ} \mathrm{C}$ for electrons

is obtained in this work via energy calibration at different temperatures in 'pure' LAB based liquid scintillator (Sect. 2.3). This is in the same order of magnitude as the observation by Xia et al. [5], showing diagrams which correspond to coefficients of $\approx-0.30 \% /{ }^{\circ} \mathrm{C}$. Their setup is well designed and thus their result seems trustworthy and find more or less a confirmation with this independent study. Although there are more studies to mention here, namely those by Seliger and Ziegler [6], Laustriat and Coche [8], Homma et al. [12] and Buontempo [13], all of these have significant problems either in their measurement concept or present their data in way that does not allow for a direct comparison. Nevertheless, all of those publications (except [6]) indicate and show a significant increase in electron generated light output at lower temperatures.

For $\alpha$-particles previous measurement data is even more scarce, only Homma et al. [10,12] present pulse-height spectra from ${ }^{241} \mathrm{Am}$ at different temperatures. Unfortunately they do not analyse these spectra quantitatively but show only very coarse diagrams. A very rough calculation from a 'by hand' analysis of the presented spectra, using only two data points for ${ }^{241} \mathrm{Am}$ in a PPO-benzene mixture, results in $\approx-0.5 \% /{ }^{\circ} \mathrm{C}$. In this work, the combined average temperature coefficient for all energies, including the values for ${ }^{222} \mathrm{Rn},{ }^{218} \mathrm{Po},{ }^{214} \mathrm{Po}$ and ${ }^{147} \mathrm{Sm}$, is

$$
(-0.35 \pm 0.03) \% /{ }^{\circ} \mathrm{C} \text { for } \alpha \text {-particles, }
$$

which is in the same order of magnitude again and confirms the overall trend. It must be pointed out that it is difficult to combine the coefficients originating from two scintillator types. While the diffusion of radon atoms does not involve 


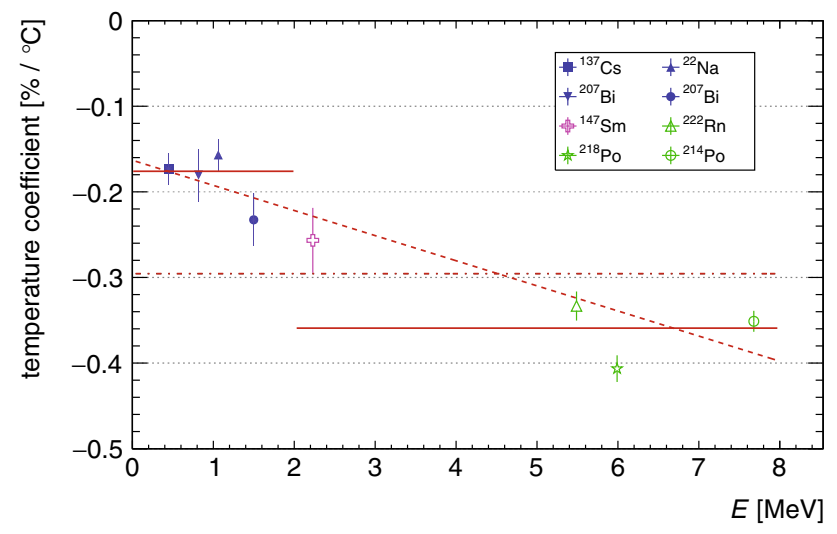

Fig. 23 Temperature coefficients for four gamma-ray emitting isotopes (blue, filled data points) and four $\alpha$-emitters (green hollow points, pink for samarium) in LAB based liquid scintillator. Model testing has been done by fitting a global constant (red dash-dotted line) two separate constants (red solid lines) and a linear function (red dashed line)

any significant change in the chemistry of the liquid scintillator, the metal loading technique does. Therefore, a comparison of the independent temperature coefficients is sensible and can be done by analysing these values energy dependent.

Figure 23 illustrates the combined data for electrons (generated by gamma-rays in 'pure' scintillator, Sect. 2.3) in blue and for $\alpha$-particles in radon- and samarium loaded LAB mixtures (green and pink, hollow symbols). The first observation is the already mentioned overall trend of slightly negative temperature coefficients of less than one percent per degree Celsius. Secondly, it is clearly visible that the samarium value (pink data point) deviates from the other $\alpha$-isotope values, measured in the radon loaded scintillator, and is connected to a much larger uncertainty. This can partly be explained by its position at lower energies, but might also have its origin in the fact that it is measured in another scintillator mixture, which is already expressed in the worse optical properties and worse energy resolution.

Concerning a possible energy dependence, three very simple models have been tested. Fitting a global constant describes the data very badly ( $\mathrm{p}$-value $<10^{-10}$ ) but excludes a zero temperature coefficient already by more than $30 \sigma$. The fit value for a global constant is $(-0.29 \pm 0.01) \% /{ }^{\circ} \mathrm{C}$. A better approximation, but still very bad, is possible by assuming a linear function, which yields a $p$-value of $\sim 10^{-6}$. Out of the chosen models, a fit with separate constants for

electrons $(-0.17 \pm 0.01) \% /{ }^{\circ} \mathrm{C}$ and

alphas $(-0.36 \pm 0.01) \% /{ }^{\circ} \mathrm{C}$

yields the best result with a probability value of $6 \times 10^{-4}$. One reason for the overall bad results in approximating an analytical model is the position and large uncertainty of the ${ }^{147} \mathrm{Sm}$ data point. With these few data points, a final con- clusion seems difficult and none of the three simple models can describe the data well. Judging purely from the numbers, the two constants model has a slight preference. As it is very difficult to measure $\alpha$ sources at even lower energies (due to the ten-fold quenching in energy), the only realistic option in clarifying this would be measuring higher energy electrons, in the range above $3 \mathrm{MeV}$. This might be achievable with high energy gamma rays or the measurement of the energy spectrum of the electrons from muon-decay, reaching up to 50 $\mathrm{MeV}$ at their upper, sharp end. The data point estimated from $\mathrm{Xia}$ et al. [5] for ${ }^{137} \mathrm{Cs}$ of $\approx-0.30 \% /{ }^{\circ} \mathrm{C}$ would push the first blue data point in Fig. 23 down quite a bit and more towards the regime of the alphas. Beyond these most simple models, it could also be possible that a linear energy dependency for electrons is realised, whereas $\alpha$-particles might experience a larger shift with a temperature dependent Birks' parameter.

Pulse shape analysis Useful insights for a physics interpretation are also provided by the comparison of the pulse shape analysis results. For this work, the temperature behaviour of averaged pulse shapes from ${ }^{147} \mathrm{Sm},{ }^{214} \mathrm{Po}$ and ${ }^{214} \mathrm{Bi}$ have been analysed. All of these are averages of a very large amount of shapes, on the order of 1 million each. They have been fitted by a two part exponential function with two separate decay constants (Eq. 2), which turned out to be the only reasonable way to approximate these shapes analytically. All three isotopes show a significant increase in the strength of the later pulse shape with lower temperatures, such that the decay constant characterising the 'slow' decay component increases. An overview of some of these values is given in Table 5. When comparing the diagrams for the relative change of the constants (Figs. 20,22) the similarities are striking. Especially the trace of the data points for the slow component seem to follow a non-linear curve, maybe quadratic or exponential. Nevertheless, the simplest linear approximation gives the following results for the slope of the slow decay constant versus temperature:

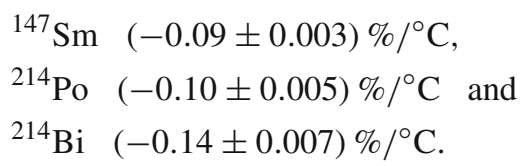

As the two $\alpha$-particles might be actually the same, the bismuth electrons seem to show an even larger increase in the slow decay time with lower temperatures. If this particle dependence is true, a lower scintillator temperature would increase pulse-shape discrimination capabilities, although an improvement on an event-by-event basis needs to be demonstrated since these differences were found in large number averages.

A final physics interpretation needs to take a lot of different information into account. Firstly an overall increase in scintillation light with lower temperatures has been reported 
Table 5 Comparison of exponential decay constants for samarium and polonium $\alpha$-isotopes and the bismuth $\beta$-decay, at room-temperature and at $-4{ }^{\circ} \mathrm{C}$

\begin{tabular}{lllll}
\hline Isotope & $\tau_{\text {fast }} @ 24{ }^{\circ} \mathrm{C}(\mathrm{ns})$ & $\tau_{\text {fast }} @-4{ }^{\circ} \mathrm{C}(\mathrm{ns})$ & $\tau_{\text {slow }} @ 24{ }^{\circ} \mathrm{C}(\mathrm{ns})$ & $\tau_{\text {slow }} @-4{ }^{\circ} \mathrm{C}(\mathrm{ns})$ \\
\hline${ }^{147} \mathrm{Sm}$ & $4.5 \pm 0.1$ & $4.5 \pm 0.1$ & $16.9 \pm 0.1$ & $17.4 \pm 0.1$ \\
${ }^{214} \mathrm{Po}$ & $4.3 \pm 0.1$ & $4.3 \pm 0.1$ & $22.8 \pm 0.1$ & $23.6 \pm 0.1$ \\
${ }^{214} \mathrm{Bi}$ & $4.2 \pm 0.1$ & $4.2 \pm 0.1$ & $20.0 \pm 0.1$ & $20.9 \pm 0.1$ \\
\hline
\end{tabular}

throughout literature as well as it has been seen in this work. Partly this effect can be explained by the fact that at lower temperatures vibrational modes of the molecules are less excited. A linear behaviour is likely and hints are found that $\alpha$-particles and electrons have different temperature coefficients. Secondly, seemingly so far no other study has had the chance to analyse the pulse shapes of these radiations in liquid scintillator at different temperatures. This work found that the slow component of an exponential decay of the scintillation is stronger pronounced at lower temperatures. As the triplet lifetime of benzene matches this slow decay component well [21-24], this result can be seen as an indication that either more triplet states are populated or that the radiative de-excitation of those is more efficient at lower temperatures.

The increased population would fit into the theory of Birks and Conte $[9,25]$, who proposed that the energy transfer between solvent-solvent and solvent-solute molecules is mainly due to excimer formation. As excimer formation is enhanced at lower temperatures and higher viscosity, a higher light yield at lower temperatures would fit into this picture. But Mathad and Umakantha [26] disfavoured this explanation convincingly in their study. Considering all the presented arguments, it seems likely that the temperature quenching effect is mainly due to more efficient triplet de-excitation as non-radiative paths are increased with higher temperature, such as collisional processes.

Acknowledgements We like to thank Andreas Hartmann and David Weinberger for their great help with hardware and pulser electronics, as well as the Radiation Physics group at the IKTP for kindly providing the radon source. This material conducted at the Brookhaven National Laboratory is based upon work supported by the U.S. Department of Energy, Office of Science, Office of Nuclear Physics.

Open Access This article is distributed under the terms of the Creative Commons Attribution 4.0 International License (http://creativecomm ons.org/licenses/by/4.0/), which permits unrestricted use, distribution, and reproduction in any medium, provided you give appropriate credit to the original author(s) and the source, provide a link to the Creative Commons license, and indicate if changes were made.

Funded by SCOAP ${ }^{3}$.

\section{Appendix: Alternative analysis: radon loaded LAB}

This appendix section shall illustrate an alternative approach to analysing the data obtained with the radon loaded LAB mixture (see Sect. 3.2). In the relevant part of the ${ }^{222} \mathrm{Rn}$ decay

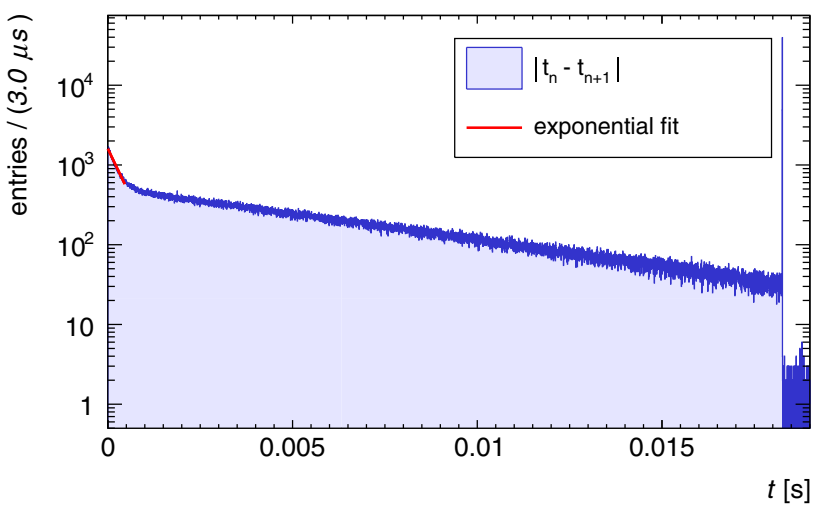

Fig. 24 Spectrum of time difference between two events in radonloaded LAB. The peak at $18 \mathrm{~ms}$ is caused by the LED pulser, marking the longest possible time difference. Events right of this structure are just possible due to the finite dead-time of the FADC. The change in the slope at the very left side is due to the ${ }^{214} \mathrm{Bi}^{-214} \mathrm{Po}$ coincidence, which is fitted with an exponential function (red solid line)

chain, the ${ }^{214}$ Po decay has very short half-life of $164.3 \mu \mathrm{s}$, compared to the other isotopes. This is an opportunity for a time coincidence analysis, which will be used as a first cut in this alternative analysis. A closer look at the times in between two events is insightful, this spectrum from one measurement is shown in Fig. 24. Theoretically, the timedifference distribution between ${ }^{214} \mathrm{Bi}$ and the subsequent ${ }^{214} \mathrm{Po}$ event should be an exponential with a decay constant of $4219 \mathrm{~s}^{-1}$. When looking for this structure in the time - difference spectrum in Fig. 24, it can be found at the left end of the spectrum in the interval $[0 \mu \mathrm{s}, 500 \mu \mathrm{s}]$. Events with the less steep slope in centre belong to the other decays with smaller decay constants and therefore longer half-lives.

It is clearly visible that the spectrum is continuous and that the overall activity is quite high, which is not beneficial for this kind of analysis. This high activity of about $1 \mathrm{kBq}$ at the beginning of the measurements was chosen due to practical reasons. For each temperature point some hours are needed to perform the measurements and changing the temperature of the liquid scintillator and the detector, so that about one temperature per day could be investigated. Thus, making 10 measurements took about 2 weeks, which is just in the range of the ${ }^{222} \mathrm{Rn}$ half-life of $T_{1 / 2}=3.82 \mathrm{~d}$.

This relatively high activity mainly disturbs the time coincidence between the polonium and polonium alpha, which expresses itself in a 'contamination' with background events in the energy spectra after a time cut is applied. For this reason 


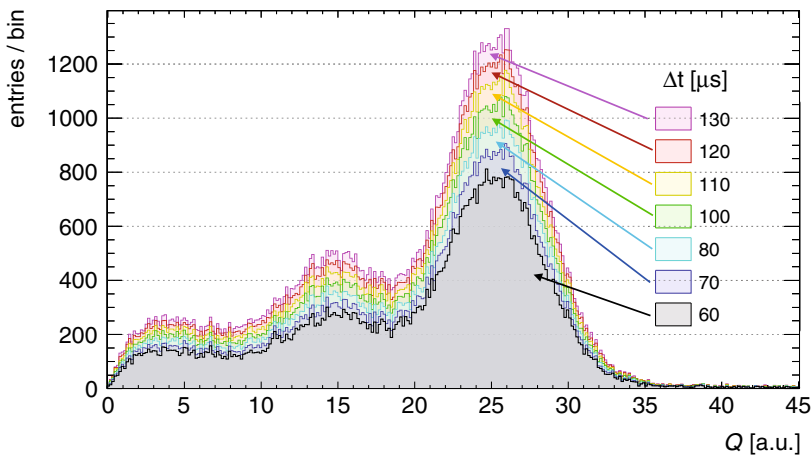

Fig. 25 Charge spectra from radon-loaded LAB, populated with events that are falling into different time windows, ranging from $60 \mu \mathrm{s}$ to $130 \mu \mathrm{s}$. The ${ }^{214} \mathrm{Po} \alpha$-peak is clearly pronounced, but also background events from the double- $\alpha$ feature and the $\beta$-spectra are still visible to the left. The $70 \mu$ s cut has the best peak to background ratio

a heuristic approach for the length of the coincidence time window is chosen. In Fig. 25, energy spectra of the second event (supposedly from ${ }^{214} \mathrm{Po}$ ) are plotted, dependent on different coincidence time windows. The $70 \mu$ s spectrum shows the best peak to background ratio, and was hereafter chosen as time cut to isolate the ${ }^{214} \mathrm{Bi}^{2}{ }^{214} \mathrm{Po}$ coincidence. Although the ${ }^{214} \mathrm{Po} \alpha$-peak is nicely pronounced and isolated with this first cut, still some events from the $\beta$-spectra and the other $\alpha$ events are 'leaking' into these spectra.

To further improve this selection, a second cut is applied, this time on the energy of either the first or the second event in the coincidence, to clean up the spectra of the other event, respectively.

To improve the ${ }^{214} \mathbf{B i} \beta$-spectra, the following selection of events is performed:

1. events with a time difference of $\leqq 70 \mu$ s

2. first event of those pairs is taken, if the second event lies within the $[817,1170] \mathrm{keV}$ energy window (the ${ }^{214} \mathrm{Po} \alpha$ peak)

The effect of this is demonstrated in Fig. 26. Although the actual signal sacrifice is large, the improvement on the shape and the strong suppression of disturbing $\alpha$-events make this cut still useful. For $\beta$-spectra it is difficult to quantify any small change of light output with temperature change, since they do not represent a steep, peak-like structure. A visual inspection reveals no visible change in the $\beta$-spectra with changing temperature. Nevertheless, an analysis of the pulse shapes indicates also a temperature dependence for the decay constants of the betas, see Sect. 3.3.

A similar event selection can be performed to enhance the

${ }^{214}$ Po spectra:

1. events with a time difference of $\leqq 70 \mu \mathrm{s}$

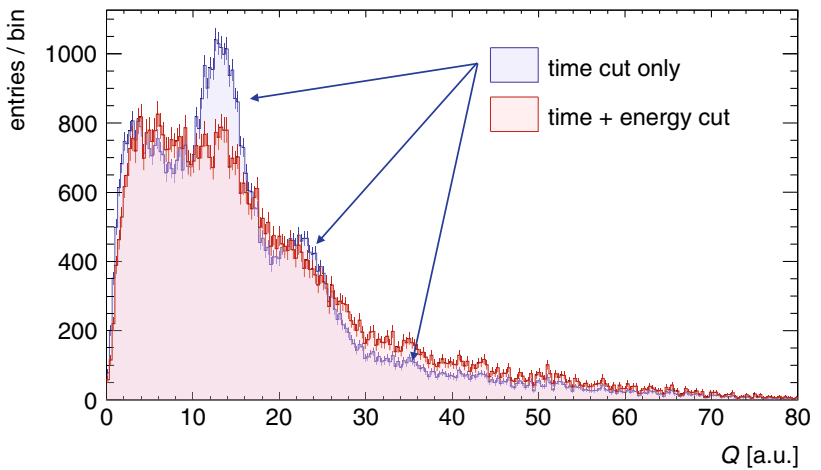

Fig. 26 Comparison of charge spectra obtained from radon loaded $\mathrm{LAB}$, when applying only time cuts (blue) and constraining also the energy of the following event to the energy of ${ }^{214} \mathrm{Po}$ (red). The peak-like features in the blue spectrum are effectively suppressed when applying the energy cut. Since signal sacrifice is large, the red spectrum is normalised to the area of the blue, for better comparison

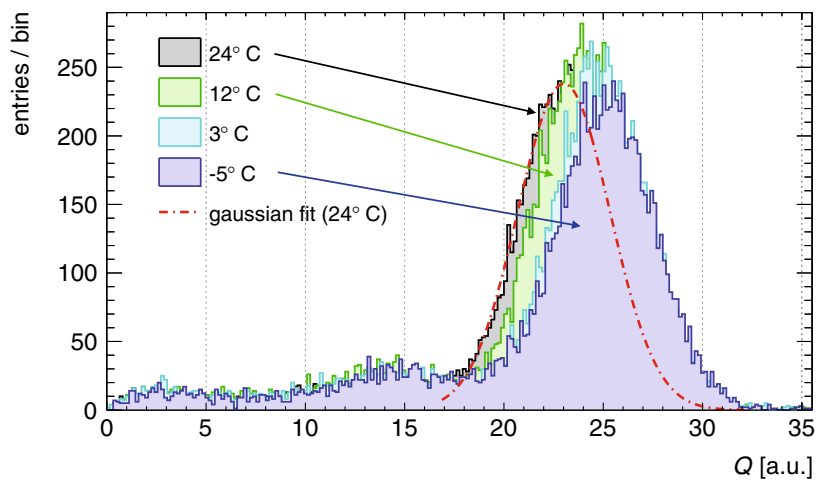

Fig. 27 Comparison of charge spectra from radon-loaded LAB $(+5 \mathrm{~g} / \mathrm{l} \mathrm{PPO}+20 \mathrm{mg} / \mathrm{l}$ bis-MSB$)$ at different temperatures, time and energy cuts applied, as well as PMT sensitivity correction. The ${ }^{214} \mathrm{Po}$ $\alpha$-peak is clearly pronounced a well Gaussian shaped. The red dashdotted line shows the Gaussian fit to the room-temperature spectrum

2. second event of those pairs is taken, if the first event lies above an energy of $0.99 \mathrm{MeV}$ (end-point energy of the ${ }^{214} \mathrm{~Pb} \beta$-spectrum)

Again, despite a large signal loss, the effect of reducing background events that disturb the shape and therefore also the position of the $\alpha$-peak, is worthwhile. Beside the greatly improved signal to background ratio (compare Fig. 25 vs. Fig. 27), also the fit range uncertainty decreased from $1.7 \%$ to $0.3 \%$. This is already referring to the final analysis of the peak positions, that again is performed in a two step fit procedure. As it has been done for the analysis of the samarium loaded LAB, here again a first standard Gaussian fit determines the rough peak position. Then, in a second step, the mean and sigma from the first fit is extracted to perform the actual Gaussian fit in the $2 \sigma$ range around this mean. Table 6 gives an exemplary overview of the uncertainty val- 
Table 6 List of uncertainties considered in the analysis of the ${ }^{214} \mathrm{Po}$ $\alpha$-peak at room-temperature $\left(24.62 \pm 0.04^{\circ} \mathrm{C}\right)$ in radon loaded $\mathrm{LAB}$

\begin{tabular}{ll}
\hline Uncertainty & Value (\%) \\
\hline Fit & 0.15 \\
Fit range & 0.3 \\
Pulser stability & 0.35 \\
Variance between rep. measurements & 0.9 \\
Sum & $\mathbf{1 . 7}$ \\
\hline
\end{tabular}

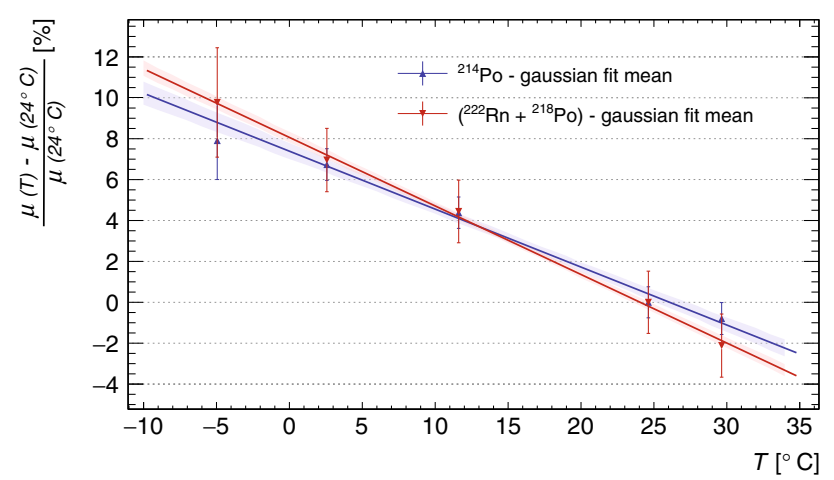

Fig. 28 Shift of the mean values from fitting the ${ }^{214}$ Po $\alpha$-peak, after applying time and energy cuts (as in Fig. 27), in blue. And from fitting the double $\alpha$-feature of ${ }^{222} \mathrm{Rn}$ and ${ }^{218} \mathrm{Po}$, in red. Each temperature point is the average of the repeated individual measurements and these are normalised to the room-temperature value at $(24.5 \pm 0.04){ }^{\circ} \mathrm{C}$. The shaded areas mark the $1 \sigma$ uncertainty band from the linear fit

ues for the analysis of the first room-temperature measurement.

An illustration of the effect of the temperature changes on the $\alpha$-peak position can be found in Fig. 27, the PMT sensitivity correction is already applied. Also a graphical representation of the fit results versus temperature is given in Fig. 28.

In a last variation of the analysis, a fit to the double $\alpha$-feature of ${ }^{222} \mathrm{Rn}$ and ${ }^{218} \mathrm{Po}$ is performed, mainly for comparison and cross-check. This time, events that fall into the $70 \mu \mathrm{s}$ coincidence time window of the ${ }^{214} \mathrm{Bi}^{2}{ }^{214} \mathrm{Po}$ time window, are excluded from the analysis. This suppresses the ${ }^{214} \mathrm{Po} \alpha$ peak very effectively as well as the background contribution of the ${ }^{214} \mathrm{Bi} \beta$-spectrum. Thus the shape and the position of the double $\alpha$-peak is less influenced by the tail of the higher energy $\alpha$ - and $\beta$-decays. This time, only one single Gaussian is fitted to the double $\alpha$-feature, though in the same manner as before. That means a first fit to roughly identify peak position and width and then a second more refined approximation. Since there is still one $\beta$-spectrum in the background, the uncertainties are much larger for this rough and simple analysis. Nevertheless the results, also shown in Fig. 28, provide a cross-check and comparison to the outcome of the other analyses and are agreeing well within the error margins.

\section{References}

1. F. An, J. Bai, A. Balantenkin et al., The Daya Bay Collaboration, Phys. Rev. Lett. 108(17) (2012)

2. J. Ahn, S. Chebotaryov, J. Choi et al., The RENO Collaboration, Phys. Rev. Lett. 108(19) (2012)

3. S. Andringa, E. Arushanova, S. Asahi et al., The SNO+ Collaboration, Adv. High Energy Phys. 2016(9) (2016)

4. F. An, G. An, Q. An, V. Antonelli et al., The JUNO Collaboration, J. Phys. G 43(3) (2016)

5. D.M. Xia, B.X. Yu et al., Chin. Phys. C 38(11), 116001 (2014)

6. H.H. Seliger, C.A. Ziegler, in Nucleonics, vol. 14 (1956), p. 49

7. G. Laustriat, A. Coche, J. de Phys. et le Radium 19(12), 927 (1958)

8. G. Laustriat, A. Coche, J. de Phys. et le Radium 21(5), 487 (1960)

9. J.B. Birks, The theory and practice of scintillation counting (Pergamon Press Ltd., Oxford, 1964)

10. Y. Homma, Y. Murase, M. Ishii, J. Radioanal. Nucl. Chem. Lett. 5(93), 263 (1985)

11. Y. Homma, Y. Murase, M. Ishii, J. Radioanal. Nucl. Chem. Lett. 5(95), 281 (1985)

12. Y. Homma, Y. Murase, K. Sonehara, Appl. Radiat. Isot. 38, 91 (1987)

13. S. Buontempo, F. Galeazzi et al., Nucl. Instr. Meth. A 425, 492 (1999)

14. T. Hakamata, Photomultiplier tubes, 3rd edn. (Hamamatsu Photonics K. K., Japan, 2007)

15. S. Flyckt, C. Marmonier, Photomultiplier tubes: principles and applications (Philips Photonics, Brive, 2002)

16. O. Klein, T. Nishina, Zeitschrift für Physik A 52, 852 (1929)

17. R. Firestone, S. Chu, L. Ekström. Website for the table of radioactive isotopes (1999). http://ie.lbl.gov/toi/. Accessed 8 Mar 2016

18. K. Kossert, G. Jörg et al., Appl. Radiat. Isot. 67(9), 1702 (2009)

19. H. Wilsenach, K. Zuber et al., Phys. Rev. C 95, 034618 (2017)

20. B.V. Krosigk, S. Hans, A.R. Junghans, et al., Eur. Phys. J. C 76(109) (2016)

21. R.B. Cundall, P.A. Griffiths, Trans. Faraday Soc. 61, 1968 (1965)

22. R.B. Cundall, W. Tippett, Trans. Faraday Soc. 66, 350 (1970)

23. M. Li, Z. Guo et al., Nucl. Instr. Meth. A 830, 303 (2015)

24. H. O'Keeffe, E. O'Sullivan, M. Chen, Nucl. Instr. Meth. A 640 , $119(2011)$

25. J.C. Conte, J.B. Birks, G. Walker, J. Phys. B: At. Mol. Phys. 1, 934 (1968)

26. R. Mathad, N. Umakantha, Phys. Lett. A 108(3), 167 (1985) 